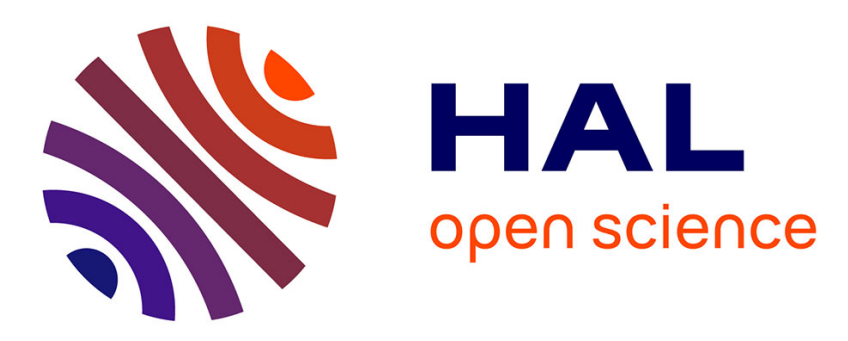

\title{
Impacts of discretization error, flow modeling error and measurement noise on inverse transport-diffusion-reaction in a T-junction
}

\author{
Julien Waeytens, Patrice Chatellier, Frédéric Bourquin
}

\section{To cite this version:}

Julien Waeytens, Patrice Chatellier, Frédéric Bourquin. Impacts of discretization error, flow modeling error and measurement noise on inverse transport-diffusion-reaction in a T-junction. Journal of Fluids Engineering, 2017, 139 (5), 32 p. 10.1115/1.4035806 . hal-01480219

\author{
HAL Id: hal-01480219 \\ https://hal.science/hal-01480219
}

Submitted on 4 Jan 2019

HAL is a multi-disciplinary open access archive for the deposit and dissemination of scientific research documents, whether they are published or not. The documents may come from teaching and research institutions in France or abroad, or from public or private research centers.
L'archive ouverte pluridisciplinaire HAL, est destinée au dépôt et à la diffusion de documents scientifiques de niveau recherche, publiés ou non, émanant des établissements d'enseignement et de recherche français ou étrangers, des laboratoires publics ou privés. 


\title{
Impacts of discretization error, flow modeling error and measurement noise on inverse transport-diffusion-reaction in a T-junction
}

\author{
Julien Waeytens \\ Researcher \\ Université Paris-Est, IFSTTAR \\ 14-20 boulevard Newton \\ Marne-la-Valle, F-77447 \\ France \\ Email: julien.waeytens@ifsttar.fr
}

\author{
Patrice Chatellier \\ Director of Laboratory \\ on Instrumentation, \\ Scientific Informatics and Simulation \\ Université Paris-Est, IFSTTAR \\ 14-20 boulevard Newton \\ Marne-la-Valle, F-77447
}

France

Email: patrice.chatellier@ifsttar.fr

\author{
Frédéric Bourquin \\ Head of Components and Systems Department \\ Université Paris-Est, IFSTTAR \\ 14-20 boulevard Newton \\ Marne-la-Valle, F-77447 \\ France \\ Email: frederic.bourquin@ifsttar.fr
}

\begin{abstract}
By combining a physical model and sensor outputs in an inverse transport-diffusion-reaction strategy, an accurate concentration cartography may be obtained. The paper addresses the influence of discretization errors, flow uncertainties and measurement noise on the concentration Paper number: FE-16-1416, Corresponding author: Waeytens, Page 1
\end{abstract}


Copyright (c) 2 jield reconsiruction process. We consider a key element of a drinking water network, i.e. a pipe junction, where Reynolds and Peclet numbers are approximately 2000 and 1000 respectively. We show that a $10 \%$ error between the reference concentration field and the reconstructed concentration field may be obtained using a coarse discretization. Nevertheless, to keep the error below $10 \%$, a fine concentration discretization is required. We also details the influence of the flow approximation on the concentration reconstruction process. The flow modeling error obtained when the exact Navier-Stokes flow is approximated by a Stokes flow may lead to a $40 \%$ error in the reconstructed concentration. However if the flow field is obtained from the full set of Navier-Stokes equations, we show that the error may be less than 5\%. Then, we observe that the quality of the reconstructed concentration field obtained with the proposed inverse technique is not deteriorated when sensor outputs have a normal distribution noise variance of few percents. Lastly, a good engineering practice would be to stop the reconstruction process according to an extended discrepancy principle including modeling and measurement errors. As shown in the article, the quality of the reconstructed field declines after reaching the threshold of the modeling error.

\section{Introduction}

In drinking water networks, the water quality has to be guaranteed to the users by the operator. Hence, chlorine is injected into the network to maintain the water quality between the water treatment plant and the end-users. The chlorine eliminates microorganisms and biofilm on the pipe walls at a reasonable cost. According to European standards, the chlorine concentration must be within a predefined range in drinking water networks. The lower limit is associated to chlorine disinfection purposes whereas the higher limit is defined as regards of the water taste. To palliate the lack of measurements in drinking water networks, physical models based on partial differential equations can be employed to predict the time evolution of the water quality at any point of the water network.

The combination of physical models and sensor outputs via data assimilation techniques was previously used to reconstruct the water flow $[1,2]$, to cartography the air quality [3] or the water quality $[4,5]$. The optimal control theory [6] may be applied to reconstruct the chlorine concentration in water networks. In this framework, the reconstruction problem is rewritten as an optimization problem in which Paper number: FE-16-1416, Corresponding author: Waeytens, Page 2 
Copyright the boundary conditions, i.e. the control parameters, are sought to minimize a data misfit functional.

This minimization problem can be solved by iterative gradient methods. The functional gradient can be computed at a lower cost than classical finite differences using the adjoint state. When focusing on the dispersion of chlorine concentration, both direct and adjoint problems are linear transport-diffusionreaction equations. Contrary to the direct problem, the adjoint problem is backward in time, i.e. null final condition, and virtual chlorine is injected at the sensor location. Indeed, in the adjoint problem, the chlorine injection amplitude is given by the gap between the measured and the simulated chlorine. Let us note that the adjoint framework is used in many domains: sensitivity analysis [7], error estimation on a quantity of interest [8,9], goal-oriented model updating [10], optimal sensor placement [5] and shape optimization [11-13].

In the water quality applications, two time scales can be distinguished for the inverse problems. Model parameters such as the kinetic parameter may be updated $[14,15]$ once a day due to their low time evolution. However, the reconstruction of the chlorine concentration have to be performed several times per hour in real-time. Two ways can be investigated to get a real-time reconstruction. Firstly, real-time computations can be achieved using reduced order methods $[16,17]$. It consists to perform the expensive computation in an offline stage. Hence, sophisticated models such as computational fluid dynamics (CFD) models which are employed in aerodynamic [18] and air quality applications [19] can be considered. Secondly, instead of using complex models, we can use simpler flow and dispersion models. Simplified one-dimensional models are traditionally implemented in drinking water software such as EPANET [20] to simulate the flow and the propagation of the chlorine concentration. Nevertheless, these simplified models may not be suitable for some key elements of the water networks, especially in junctions where the hypothesis of perfect and instantaneous mixing may not be valid for laminar flow encountered in distribution mains [21]. Consequently, one can consider hybrid model approaches, previously applied to biomechanics applications [22] combining coarse model in straight pipes and refined model in junctions.

In the present article, our main objective is to analyze the influence of several factors on the concentration reconstruction process. The inverse transport-diffusion-reaction method and the application to a T-junction are presented in Section 2. To save computation time in the concentration reconstruction process, one can solve direct and adjoint problems using coarse discretizations. Hence, the influence 
Copyright of the concentration discretization errors is investigated in Section 3. Then, noting that the flow is not well-known in general in water pipe applications [23], the influence of the flow approximation on the quality of the reconstructed concentration is studied in Section 4. In particular, the influence of flow uncertainties due to modeling error is studied. In Section 5, both influences of discretization errors and modeling errors on the reconstructed concentration field are considered. In the last section we analyze the effect of measurement noise on the reconstruction process. For instance, in the water pipe study, if the flow field is properly known, we show that the error obtained in the concentration field may be less than 5\%. However, if an approximate flow field is used in the reconstruction process, a $40 \%$ error in the concentration field may be obtained. Finally, concerning the robustness of the inverse technique, we show that the quality of the reconstructed chlorine concentration does not deteriorate when considering corrupted data with a noise variance of few percents. These may be met in the case of measurement error.

\section{Inverse transport-diffusion-reaction method applied to the concentration field reconstruction in a T-junction}

\subsection{Summary of the inverse transport-diffusion-reaction method}

To reconstruct the space-time cartography of a concentration field for drinking water purposes, the authors have previously proposed in [5] an inverse strategy combining linear transport-diffusion-reaction equations in $2 \mathrm{D}$ and concentration sensor outputs. In the present section, only the outlines of the method are given. The full details of the inverse strategy can be found in [5].

The considered inverse method is based on the optimal control theory [6]. The control parameter $\mathbf{u}$, which correspond herein to concentration boundary conditions, is sought such as it minimizes the data misfit functional $J$ defined by:

$$
J(\mathbf{u})=\frac{1}{2} \sum_{j=1}^{n_{s}} \int_{0}^{T}\left(\int_{\Omega} \psi_{j}^{r} C(\mathbf{u}) d \Omega-C_{j}^{m e s}\right)^{2} d t+\alpha R(\mathbf{u}, C)
$$

where $C$ denotes the concentration field satisfying 2D linear transport-diffusion-reaction equations, $\psi_{j}^{r}$ Paper number: FE-16-1416, Corresponding author: Waeytens, Page 4 
Copyright (resp. $C_{j}^{17}$ ) is an extracting function (resp. the measured concentration) associated with the $j^{\text {th }}$ sensor at $\mathbf{x}_{\mathbf{j}}, n_{s}$ is the number of chlorine sensors and $\alpha R(\mathbf{u}, C)$ is a regularization term.

As we numerically observe that the chlorine reconstruction inverse problem is not ill-posed, the regularization term does not play a significant role. Consequently, the regularization parameter $\alpha$ is taken to zero. The influence of the regularization on the quality of the reconstructed filed lies out of the scope of the article.

To solve the optimization problem associated to the chlorine reconstruction, i.e. minimization of the data misfit functional $J$, a gradient-based iterative method is used. At each iteration, the different steps can be summarized as follows:

1. solve the linear transport-diffusion-reaction equations, also called "direct problem", considering the concentration boundary conditions $\mathbf{u}_{\mathbf{o l d}}$ determined at the previous iteration;

2. solve the adjoint problem, i.e. linear transport-diffusion-reaction equations backward in time where virtual chlorine is injected at the sensor location. The chlorine injection amplitude is given by the gap between the measured and the simulated chlorine;

3. compute the gradient $\nabla \mathbf{J}$ of the functional at a low computational cost using the direct and the adjoint numerical solutions;

4. determine the optimal descent step $\gamma$ and get the updated control parameter

$$
\mathbf{u}_{\text {new }}=\mathbf{u}_{\text {old }}-\gamma \nabla \mathbf{J}
$$

Let us note that at each iteration the space-time cartography of the concentration field is updated by computing the direct problem. In the next sections, we analyze the quality of the reconstructed concentration field obtained at each iteration.

The authors verified the ability of the considered gradient-based method to solve optimization problems for the reconstruction of the flow in drinking water networks [2] and for the reconstruction of the chlorine concentration in water pipes [5].

Paper number: FE-16-1416, Corresponding author: Waeytens, Page 5 


\subsection{Description of the study case: a water pipe T-junction}

In the design of water networks one can distinguish two kinds of pipes, "transport mains" and "distribution mains". The first ones are build of large pipes which usually don't supply any costumer. The second ones involve much smaller pipes connecting the "transport mains" to the end-user. The design of these pipes diameter is mainly based on the liquid retention time which must be limited in order to avoid the development of bacteria. This limitation leads to smaller diameters of the pipes. This result makes networks cheaper to build but leads to higher pressure drops. Furthermore in case of fire, the pipes diameter of the network should not be too small in order to meet fire flow demand. Obviously this demand is mandatory and is much higher than the domestic demand [24]. All these considerations lead to areas of the networks where typical velocities can be very low (less than $1 \mathrm{~cm} / \mathrm{s}$ ) and change rapidly from one second to the next [21].

Our objective is to reconstruct the chlorine concentration in "distribution mains" for a given flow. Let us note that the authors have previously studied in [2] the reconstruction by an inverse technique of the flow encountered in the "distribution mains" of a drinking water network. It has been applied to the T-junction and to a part of a French water network.

Therefore we have chosen to study a T-junction represented in Fig. 1. This is one of the key elements of a water network, and it is simple enough to enable an easy understanding the complexity of the flow and concentration fields in the network. To study this T-junction we first consider a constant flow, and then a fluctuating flow (Section 5). In order to have a better understanding of the reconstruction technique we first deal with simplified boundary velocities (see Fig. 2) and Reynolds numbers less than $2 \times 10^{3}$. This enables us to compute all numerical solutions using the finite element code FreeFem++ [25]. The reference flow chosen for our study satisfies the Navier-Stokes and incompressibility equations. On the boundary $\partial_{d} \Omega$ of $\partial \Omega$, the velocity vanishes. On the boundary $\partial_{c} \Omega$ of $\partial \Omega$, velocities $\mathbf{v}_{\mathbf{c}}$ are prescribed. The velocities at the boundaries, named $\left(\mathbf{v}_{\mathbf{c}_{1}}, \mathbf{v}_{\mathbf{c}_{2}}, \mathbf{v}_{\mathbf{c}_{3}}\right)$, are normal to the boundaries and their shape $g_{i}(\mathbf{x})$ is considered parabolic (Poiseuille flow):

$$
\mathbf{v}_{\mathbf{c}_{\mathbf{i}}}(\mathbf{x}, t)=v_{c_{i}}^{\max }(t) g_{i}(\mathbf{x}) \mathbf{n}_{\mathbf{i}}, i \in\{1,2,3\}
$$

Paper number: FE-16-1416, Corresponding author: Waeytens, Page 6 
The transport-diffusion-reaction equation is implemented with the following parameters so that it includes the full complexity of the modeled phenomena. The diffusion coefficient is taken equal to that of chlorine in water $v=10^{-5} \mathrm{~m}^{2} / \mathrm{s}$ leading to a Peclet number of approximately $10^{3}$. For the kinetics of the reaction we have implemented a first order reaction, meaning that the kinetics is equal to $k C$, where $\mathrm{k}$ is the kinetic coefficient [20]. From analyses of treated water in U.S. drinking water networks, the kinetic coefficient ranges from $0.08 \mathrm{day}^{-1}$ to $18 \mathrm{day}^{-1}$ [26]. In the present article, we have considered a kinetic coefficient of $k=5 d a y^{-1}$ that is in the upper end of the range. A value as high as that may be found when readily biodegradable pollutant is being degraded by chlorine. On the boundary $\partial_{d} \Omega$, we do not introduce the wall reaction which might be the case when no biofilm is present. Lastly, on the boundary $\partial_{c} \Omega$, the spatial shape of the control concentration $\mathbf{U}=\left(U_{1}, U_{2}, U_{3}\right)$, is considered constant:

$$
U_{i}(\mathbf{x}, t)=u_{i}(t) f_{i}(\mathbf{x}) \text { with } f_{i}(\mathbf{x})=1, i \in\{1,2,3\}
$$

When considering a laminar flow in a straight pipe, let us remark that the transverse uniformity of the concentration distribution can be established for a length above $10 \mathrm{v} R^{2} / v[27]$ where $R$ (resp. v) corresponds to the radius of the straight pipe (resp. the pipe average velocity).

Using all these parameters the problem we are addressing in the following is: one seeks $\left(u_{1}(t), u_{2}(t), u_{3}(t)\right)$ using the concentration outputs from 2 sensors. In practice, the sensor outputs are pointwise and experimental. Nevertheless, the sensor outputs are obtained here numerically from the following steps. First, using a very fine discretization $\left(1.1 \times 10^{5}\right.$ dof, 2560 time steps $)$, we solve the transport-diffusionreaction problem considering the reference boundary concentrations $\left(U_{1}^{e x}, U_{2}^{e x}, U_{3}^{e x}\right)$ shown in Fig. 3 . The associated solution is $C_{e x}$. Then, we compute the simulated sensor outputs using

$$
C_{j}^{m e s}=\int_{\Omega} \psi_{j}^{r} C_{e x} d \Omega \approx C_{e x}\left(\mathbf{x}_{\mathbf{j}}\right)
$$

Paper number: FE-16-1416, Corresponding author: Waeytens, Page 7 
In the three next sections, we do not introduce noise in the simulated sensor outputs. The influence of the measurement noise on the reconstructed field is studied in the last section.

In this article, direct and adjoint problems are solved using the Characteristic-Galerkin method of order $1[28]$ and a backward Euler scheme.

For a given flow, the influence of the discretization error on the concentration reconstruction is analyzed. Then, the influence of the flow approximation is studied. To evaluate the quality of the reconstruction process, we define the following normalized errors in the space-time domain:

$$
\varepsilon_{s_{j}}=\frac{\left\|\int_{\Omega} \psi_{j}^{r} C_{h} d \Omega-C_{j}^{m e s}\right\|_{L^{2}(\Omega \times[0 ; T])}}{\left\|C_{j}^{m e s}\right\|_{L^{2}(\Omega \times[0 ; T])}}, \varepsilon_{c_{j}}=\frac{\left\|C_{h}-C_{e x}\right\|_{L^{2}\left(\partial_{c_{j}} \Omega \times[0 ; T]\right)}}{\left\|C_{e x}\right\|_{L^{2}\left(\partial_{c_{j}} \Omega \times[0 ; T]\right)}}, \varepsilon_{C}=\frac{\left\|C_{h}-C_{e x}\right\|_{L^{2}(\Omega \times[0 ; T])}}{\left\|C_{e x}\right\|_{L^{2}(\Omega \times[0 ; T])}}
$$

The normalized indicator $\varepsilon_{s_{j}}$ denotes the $j^{\text {th }}$ data misfit whereas $\varepsilon_{c_{j}}$ (resp. $\varepsilon_{C}$ ) represents the error between the exact boundary concentration and the reconstructed boundary concentration on the boundary $\partial_{c_{j}}$ (resp. the error between the exact concentration and the reconstructed concentration on the whole space-time domain).

\section{Influence of the discretization error on the concentration reconstruction}

In this section, we compare the reconstructed concentration for two discretizations:

- fine discretization (Fig. 4): $2.8 \times 10^{4}$ dof, 1280 time steps

- coarse discretization (Fig. 5): $1.9 \times 10^{3}$ dof, 256 time steps.

These discretizations are used to solve the direct and adjoint problems when considering a high kinetic coefficient $k$ of $5 d a y^{-1}$. To evaluate the discretization errors in space and in time, we compute the error estimators previously introduced in [5]. The numerical values of the error estimators are given in Eq. (7).

The combination of chlorine sensors $1 \& 2$ and the reference Navier-Stokes flow are considered. The normalized errors (6) are given in Tables 1 and 2. For both discretizations, the quality of the Paper number: FE-16-1416, Corresponding author: Waeytens, Page 8 
Copyright (Table 17 . Chlorine reconstruction for the Navier-Stokes flow - Sensors 1 \& 2 - Fine mesh $\left(2.8 \times 10^{4}\right.$ dof 1280 time steps $)$ High kinetic coefficient: $k=5 d a y^{-1}$

\begin{tabular}{cccccc}
\hline & $\varepsilon_{s_{1}}(\%)$ & $\varepsilon_{s_{2}}(\%)$ & $\varepsilon_{c 1}(\%)$ & $\varepsilon_{c 3}(\%)$ & $\varepsilon_{C}(\%)$ \\
\hline Iteration 0 & 100 & 100 & 100 & 100 & 100 \\
Iteration 1 & 18.5 & 7.9 & 22.3 & 74.6 & 27.5 \\
Iteration 2 & 4.8 & 7.9 & 13.9 & 74.5 & 23.6 \\
Iteration 4 & 2.4 & 5.2 & 11.5 & 70.5 & 21.7 \\
Iteration 8 & 1.7 & 4.0 & 9.4 & 63.9 & 19.1 \\
Iteration 16 & 1.1 & 2.8 & 7.0 & 54.5 & 15.3 \\
Iteration 32 & 0.6 & 1.6 & 4.8 & 42.5 & 10.8 \\
Iteration 64 & 0.4 & 0.8 & 3.3 & 30.1 & 7.2 \\
Iteration 128 & 0.2 & 0.4 & 2.5 & 22.6 & 5.2 \\
\hline
\end{tabular}

reconstructed concentration field at the first iteration is similar. One has an error on boundary control 1 (resp. boundary control 3) of approximately 30\% (resp. 70\%) and an error in the reconstructed concentration of $25 \%$. Nevertheless, from the $16^{\text {th }}$ iteration, we observe a significant difference in the quality of the reconstructed concentration. In fact, a $2 \%$ error in the reconstructed concentration is obtained at iteration 32 with the fine discretization whereas the error is approximately $10 \%$ for the coarse discretization. Although the data misfit tends toward 0 , the error in the reconstructed concentration can't be less than $10 \%$ when using the coarse discretization. This is caused by the discretization error. At the $4^{\text {th }}$ iteration, the error estimators in space and in time associated with the reconstructed velocity obtained with the fine and the coarse meshes are:

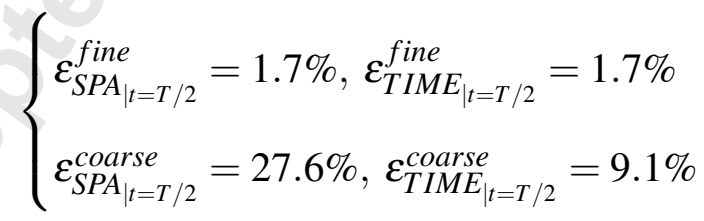

Herein, discretization errors in space and in time of about $1 \%$ allows the reconstruction of the concentration field with an error less than $5 \%$.

Concerning the computation time, on a single core $2.5-\mathrm{GHz}$ processor one needs

- $2250 \mathrm{~s}$ per iteration when using the fine discretization

Paper number: FE-16-1416, Corresponding author: Waeytens, Page 9 
Copyright (Table 2. Chlorine reconstruction for the Navier-Stokes flow - Sensors 1 \& 2 - Coarse mesh $\left(1.9 \times 10^{3}\right.$ dof 256 time steps) - High kinetic coefficient: $k=5 d a y^{-1}$

\begin{tabular}{cccccc}
\hline & $\varepsilon_{s_{1}}(\%)$ & $\varepsilon_{s_{2}}(\%)$ & $\varepsilon_{c 1}(\%)$ & $\varepsilon_{c 3}(\%)$ & $\varepsilon_{C}(\%)$ \\
\hline Iteration 0 & 100 & 100 & 100 & 100 & 100 \\
Iteration 1 & 20.9 & 9.1 & 31.7 & 72.2 & 25.7 \\
Iteration 2 & 4.7 & 4.2 & 27.3 & 74.5 & 21.1 \\
Iteration 4 & 1.9 & 3.4 & 22.2 & 71.6 & 18.5 \\
Iteration 8 & 0.8 & 1.3 & 17.8 & 68.8 & 14.8 \\
Iteration 16 & 0.6 & 0.8 & 16.8 & 57.7 & 12.3 \\
Iteration 32 & 0.2 & 0.6 & 16.3 & 53.2 & 10.7 \\
Iteration 64 & 0.2 & 0.6 & 16.0 & 55.7 & 10.1 \\
Iteration 128 & 0.1 & 0.5 & 15.7 & 60.7 & 9.9 \\
\hline
\end{tabular}

- 105 s per iteration when using the coarse discretization.

At each iteration, the most expensive calculations are the resolution of the direct problem, the resolution of the adjoint problem and the determination of the descent step by solving an additional direct problem. Let us recall that both problems are solved with the Characteristic-Galerkin method and a backward Euler scheme. As the linear system is symmetric, the Crout direct solver is employed.

To summarize, a $10 \%$ error in the reconstructed concentration can be obtained using the coarse discretization in 64 iterations, which corresponds to a $112 \mathrm{~min}$ of computation time on a single core 2.5$\mathrm{GHz}$ processor. To obtain a $3 \%$ error, one needs to use the fine discretization, which leads to a 5 times more expensive calculation. A good strategy to save computation time would be to successively use the coarse discretization and then the fine discretization. Exploring this tracle lies out of the scope of the paper.

In Tables 1 and 2, the errors on the chlorine reconstruction are obtained for a high kinetic coefficient of $5 d a y^{-1}$. To show that these results are of general relevance, i.e. not depending on the kinetic coefficient, Table 3 gives the normalized errors $\varepsilon_{s_{j}}, \varepsilon c_{j}$ and $\varepsilon_{C}$ for a low kinetic coefficient of $0.5 d a y^{-1}$ considered as a standard value in [29]. It can be noted that the numerical values of the normalized errors are similar to those of Table 1.

Paper number: FE-16-1416, Corresponding author: Waeytens, Page 10 
Copyright (fable 3 . Chlorine reconstruction for the Navier-Stokes flow - Sensors 1 \& 2 - Fine mesh $\left(2.8 \times 10^{4}\right.$ dof 1280 time steps $)$ - Low kinetic coefficient: $k=0.5 d a y^{-1}$

\begin{tabular}{cccccc}
\hline & $\varepsilon_{s_{1}}(\%)$ & $\varepsilon_{s_{2}}(\%)$ & $\varepsilon_{c 1}(\%)$ & $\varepsilon_{c 3}(\%)$ & $\varepsilon_{C}(\%)$ \\
\hline Iteration 0 & 100 & 100 & 100 & 100 & 100 \\
Iteration 1 & 18.6 & 7.9 & 22.4 & 74.5 & 27.5 \\
Iteration 2 & 4.9 & 7.9 & 13.8 & 74.4 & 23.5 \\
Iteration 4 & 2.4 & 5.2 & 11.6 & 70.4 & 21.7 \\
Iteration 8 & 1.7 & 4.0 & 9.4 & 63.8 & 19.0 \\
Iteration 16 & 1.1 & 2.6 & 7.1 & 54.5 & 15.2 \\
Iteration 32 & 0.8 & 1.3 & 4.8 & 42.4 & 10.8 \\
Iteration 64 & 0.4 & 0.7 & 3.3 & 30.0 & 7.1 \\
Iteration 128 & 0.2 & 0.4 & 2.5 & 22.7 & 5.2 \\
\hline
\end{tabular}

In the following sections, we only consider the case of a high kinetic coefficient.

\section{Influence of flow uncertainty on the reconstructed concentration field}

In the previous sections, we assume that the reference Navier-Stokes flow was exactly known. Nevertheless, this is not the case most of the time in water network applications. Indeed, approximate flow velocities are commonly used to predict the evolution of the chlorine concentration [20]. In this section, our main objective is to evaluate the influence of the flow uncertainty on the quality of the reconstructed concentration field. Two different types of approximate flow are considered in the transport-diffusionreaction equations. The first flow type corresponds to the exact flow with a perturbation part. The second flow type is the flow obtained by means of velocity reconstruction process [2].

To evaluate the quality of the approximate velocity $\mathbf{v}_{\mathbf{h}}$, a similar normalized error in the space-time domain is introduced:

$$
\varepsilon_{v}=\frac{\left\|\mathbf{v}_{\mathbf{h}}-\mathbf{v}_{\mathbf{e x}}\right\|_{L^{2}(\Omega \times[0 ; T])}}{\left\|\mathbf{v}_{\mathbf{e x}}\right\|_{L^{2}(\Omega \times[0 ; T])}}
$$

Paper number: FE-16-1416, Corresponding author: Waeytens, Page 11 


\section{Copyright $4.1^{201}$ Using the exact velocity field with a perturbation part}

For simplicity, let us first consider the following approximate velocity:

$$
\mathbf{v}_{\mathbf{h}}=(1+\varepsilon) \mathbf{v}_{\mathbf{e x}}
$$

One can notice that the perturbation parameter $\varepsilon$ exactly corresponds to the normalized error $\varepsilon_{v}$ defined in Eq. (8).

The concentration field is then reconstructed for the 2D study case (Fig. 1) using the above approximate flow. The fine discretization is employed to solve the transport-diffusion-reaction problems. Sensors $1 \&$ 2, presented in Fig. 1, are considered. For different values of the velocity perturbation parameter $\varepsilon$, we present the quality of the reconstructed concentration in Fig. 6. Until 16 iterations of the reconstruction process, the normalized errors between the exact concentration and the reconstructed concentration $\varepsilon_{C}$ are quite similar for the approximate flows with an uncertainty less than 5\%. For the approximate flow with an uncertainty greater than $5 \%$, one can observe that there is no use iterating many times to improve $\varepsilon_{C}$. In fact, for $\varepsilon_{v}=20 \%$, we obtain a more reliable reconstructed concentration after 1 iteration $\left(\varepsilon_{C} \approx 35 \%\right)$ than after 128 iterations $\left(\varepsilon_{C} \approx 80 \%\right)$. This result can be viewed as a generalization of the discrepancy principle to modeling errors. Indeed, the discrepancy principle initially introduced in [30] recommend the data misfit in a reconstruction process not to be less than the measurement error. Otherwise, it can lead to a wrong reconstruction field. To sum up, herein the reconstruction process has to be stopped when the data misfit reaches the modeling error induced by the flow uncertainty.

\subsection{Using a velocity field from a reconstruction process}

Before reconstructing the concentration field, the authors first determined an approximate velocity field using a similar inverse reconstruction technique based on velocity sensor outputs [2]. To quickly obtain the reconstructed velocity, two ways were investigated. The first way was to use coarse discretizations to solve the Navier-Stokes equations and its associated adjoint problem. The second way was to use a simpler flow model (as the unsteady Stokes equations or the Stokes equations) for the velocity reconstruction process, although the exact flow satisfies the Navier-Stokes equations. Therefore, one has a flow modeling error.

Paper number: FE-16-1416, Corresponding author: Waeytens, Page 12 
Copyright frit the water pipe junction (Fig. 1), the concentration field is reconstructed using these approximate flows. The fine discretization is employed to solve the transport-diffusion-reaction problems. Sensors $1 \& 2$, presented in Fig. 1, are considered. The results are presented in Fig. 7. Note that the NavierStokes flow and its very fine discretization $\left(2.5 \times 10^{5}\right.$ dof, 2580 time steps $)$ corresponds to the reference flow defined in Section 2.2. The solid line with the symbol (+) represents the evolution of the quality of the reconstructed concentration $\varepsilon_{C}$ when the reference flow $\left(\varepsilon_{v}=0 \%\right)$ is considered. Let us recall that a 5\% error in the reconstructed concentration remains, even after 128 iterations because sensors $1 \& 2$ do not allow a properly determination of boundary control 3 (see Table 1). When considering the approximate Navier-Stokes flows with $\varepsilon_{v}$ less than $5 \%$, the errors in the reconstructed concentration are observed to be similar at the first iterations. Additionally, at iteration 32, one observes a $11 \%$ error in the reconstructed concentration with the reference flow whereas there is a $16 \%$ error with the coarse Navier-Stokes flow $\left(4.4 \times 10^{3}\right.$ dof, 64 time steps). By using the unsteady Stokes model with a fine discretization $\left(6.4 \times 10^{4} \mathrm{dof}, 256\right.$ time steps) to reconstruct the Navier-Stokes flow, an error in the reconstructed velocity of $16 \%$ is obtained. For this approximate flow, it is interesting to note that the error in the reconstructed concentration can not be less than $32 \%$. This result is worth when using an approximate Stokes flow with a fine discretization. In this case, $\varepsilon_{C}$ can not be less than $38 \%$.

The approximate velocity fields employed above are shown in Fig. 8. The reference concentration field and the associated reconstructed concentrations field are given in Fig. 9 and 10. For the unsteady Stokes and the Stokes flows, we observe that the chlorine coming from the branch pipe is not identified by the reconstruction process. This may be due to the fact that the functional $J$, which involves sensors 1 and 2, is not sensitive to boundary control 3 for these two approximate flows. This work confirms several former papers $[4,21,31]$ stating that the flow needs to be highly accurate before it may be used to simulate the dispersion and decay of a residual disinfectant. In this article, we clearly quantify the quality of the reconstructed concentration field as a function of the the flow accuracy.

\section{Combining the concentration field discretization errors and the flow modeling error}

In this section, we present a low-cost strategy for the reconstruction of the concentration field. To achieve this, we still employ approximate flows that are reconstructed using simple flow models, and we Paper number: FE-16-1416, Corresponding author: Waeytens, Page 13 
Copyright use the coarse discretization, represented in Fig. 5, to solve transport-diffusion-reaction problems. As previously, sensors $1 \& 2$ are considered. The influence of the concentration field discretization and the flow modeling errors is studied on two examples. In the first example, the previous smooth boundary velocities are considered (Fig. 2). Then, we aim to reconstruct the chlorine concentration for a highlyfluctuating flow, which represents the real flow in "distribution mains" [21]. The associated boundary velocities are presented in Figure 12. In both examples, the Reynolds number is approximately $2 \times 10^{3}$.

\subsection{Smooth boundary velocity}

Herein, the exact flow is described using the Navier-Stokes equations with smooth boundary velocities (Fig. 2). The results of the concentration reconstruction are presented in Fig. 11. With an approximate flow satisfying the Navier-Stokes equations, the error in the reconstructed concentration declines until iteration 128 , then a plateau is reached. When considering the coarse Navier-Stokes flow $\left(\varepsilon_{v}=9 \%\right)$, a $15 \%$ error between the exact concentration and the reconstructed concentration remains at iteration 128. It may be due to:

- the impossibility to determine boundary control 3

- the $10 \%$ uncertainty in the velocity field

- the concentration discretization errors.

It can be noted that there is no use iterating several times when the reconstructed velocity is obtained with simpler flow models. Indeed, Fig. 11 shows that we should stop the reconstruction process after 2 iterations for the unsteady Stokes and Stokes flow models.

To conclude, one observes that an acceptable concentration can be obtained even for high discretization errors in the concentration when considering a reliable flow $\left(\varepsilon_{v}<10 \%\right)$. Using the approximate NavierStokes flows and solving the concentration problems with a coarse discretization leads to a $15 \%$ error in the reconstructed concentration. Conversely, the modeling error in the flow drastically reduces the quality of the reconstructed concentration field. Even for fine discretizations, in our study case, we are not able to identify boundary concentration 3 when using a reconstructed velocity obtained from Stokes flow model. This leads to a $39 \%$ error in the reconstructed concentration.

Paper number: FE-16-1416, Corresponding author: Waeytens, Page 14 


\section{Copyright $\mathbf{5 . 2} \mathbf{2}^{01}$ Fhuctuating boundary velocity}

In this part, we aim to reconstruct the chlorine concentration for a highly-fluctuating flow, which represents the real flow in "distribution mains". Therefore, we consider the fluctuating boundary velocities presented in Fig. 12. The Navier-Stokes equations are still considered to be the reference model. To save computation time, we reconstruct the concentration filed using the approximate flow and the coarse discretization to solve the transport-diffusion-reaction problems. The approximate flow corresponds to the reconstructed velocity field obtained using a similar inverse technique [2]. The results of the concentration reconstruction are given in Fig. 13. In the T-junction, introducing flow time fluctuations does not drastically reduce the accuracy of the reconstructed concentration. As in Fig. 11, the main aspect deteriorating the reconstructed concentration is the flow approximation using a simple model, i.e. the modeling error of the flow.

\section{Influence of the chlorine measurement error on the reconstructed concentration field}

We analyze the capacity of the inverse technique to reconstruct the chlorine concentration field using realistic chlorine sensor outputs. We still consider the 2D water T-junction and the Navier-Stokes flow represented in Fig. 2. Herein, we use the exact flow (no uncertainty) and sensors $S_{1}$ and $S_{2}$ represented in Fig. 1. In the previous sections, we considered idealized sensor outputs, i.e. without noise. To test the proposed inverse method on realistic data, the chlorine sensor outputs are corrupted with random

errors following a normal distribution $\mathscr{N}\left(\mu, \sigma^{2}\right)$. This distribution might induce non physical negative values that have to be set to zero as it will be the case for a real chlorine sensor. In the first subsection, we consider a normal distribution with a null mean value $\mathscr{N}\left(0, \sigma^{2}\right)$. In the second subsection, we focus on a normal distribution with a null variance $\mathscr{N}(\mu, 0)$ wich represents a measurement offset. In both subsections, we use the fine discretization $\left(2.8 \times 10^{4}\right.$ dof, 1280 time steps $)$ to solve the direct and adjoint problems involved in the chlorine reconstruction technique. The mesh is given in Fig. 4.

\subsection{Noise normal distribution $\mathscr{N}\left(0, \sigma^{2}\right)$ in the chlorine outputs}

In Fig. 14, the corrupted data are represented for different values of $\sigma$. In this section, we recall that the mean value of the noise normal distribution vanishes. 
Copyright (c) 20 The quadity of the reconstructed concentration field, when considering corrupted outputs, is shown

in Fig. 15. We can observe that the quality of the reconstructed concentration does not deteriorate when the normal distribution of the noise has a variance less than $10^{-5} \mathrm{~g} / \mathrm{l}$. Let us notice that a variance of $10^{-5} \mathrm{~g} / \mathrm{l}$ corresponds to $3 \%$ of the maximum chlorine concentration at sensor 1 . At the $128^{\text {th }}$ iteration of the inverse problem, one has a $5 \% L^{2}$ error between the reference and the reconstructed concentrations when we use the idealized outputs $(\sigma=0)$. This error reaches $10 \%$ when considering corrupted outputs with a noise variance of approximately $6 \%$ of the maximum chlorine concentration at sensor 1.

\subsection{Noise normal distribution $\mathscr{N}(\mu, 0)$ in the chlorine outputs}

In real process, sensors may derive with time. To evaluate the sensitivity of the chlorine reconstruction technique to the bias induced by this derivation, we have added a constant offset $\mu$ to the idealized chlorine sensor outputs. The quality of the reconstructed chlorine field at each iteration of the inverse problem is given in Fig. 16 for different values of the offset $\mu$. In this case, we can see that a $10^{-5} \mathrm{~g} / 1$ offset (3\% of the maximum chlorine concentration at sensor 1) drastically deteriorates the quality of the reconstructed chlorine concentration. Indeed, the $L^{2}$ error between the reference and the reconstructed concentrations is approximately $10 \%$ at the $128^{\text {th }}$ iteration of the inverse problem, whereas the error is $5 \%$ with a null offset. To sum up, in view of reconstructing a relevant concentration field both measurement error and modelling error have to be reduced. There is no use considering a sophisticated model in the reconstruction process if the sensor outputs are of poor quality.

\section{Conclusions}

In the present paper, we studied the reconstruction of the chlorine concentration in a water pipe junction which is a key element of a water network. To achieve this purpose, boundary controls were determined to minimize the data misfit. An iterative approach based on the resolution of an adjoint problem was employed. The main goal was to study the influence of the concentration discretization errors, the uncertainty on the flow and the measurement noise on the reconstruction process. First, to save computation time, transport-diffusion-reaction problems were solved with coarse discretizations which lead to non-negligible discretization errors. In the 2D water pipe junction, it was shown that a $10 \%$ error between the reference concentration field and the reconstructed concentration field may be

Paper number: FE-16-1416, Corresponding author: Waeytens, Page 16 
Copyright obtained using a coarse discretization. To keep the error below $10 \%$, fine concentration discretizations are preferred. Then, we confirm that an accurate chlorine concentration can not be achieved if the flow is not well determined. When considering the exact flow obtained herein from the full set of Navier-Stokes equations, we show that the error on the reconstructed concentration may be less than $5 \%$. However using an approximate flow may lead to a $40 \%$ error between the reference concentration field and the reconstructed concentration field. Finally, the robustness of the inverse technique has been shown in the water T-junction. Indeed, the quality of the reconstructed chlorine concentration is not deteriorated when considering corrupted outputs with a normal distribution noise variance of few percents. Lastly, a good engineering practice would be to stop the reconstruction process according to an extended discrepancy principle including modeling and measurement errors. As shown in the article, the quality of the reconstructed field declines after reaching the threshold of the modeling error.

\section{Acknowledgements}

This research was supported by the French Inter-Ministry Fund (FUI) within the project "Smart Water Network", which involved several partners: Advitam, Ondeo Systems (Suez Environnement), EFS, A3IP, ESIEE Paris and IFSTTAR.

\section{References}

[1] Podvin, B., Fraigneau, Y., Lusseyran, F., and Gougat, F., 2006. "A reconstruction method for the flow past an open cavity". ASME Journal of Fluids Engineering, 128, pp. 531-540.

[2] Waeytens, J., Chatellier, P., and Bourquin, F., 2015. "Inverse computational fluid dynamics: Influence of discretization and model errors on flows in water network including junctions". ASME Journal of Fluids Engineering, 137(9).

[3] Zhang, L., Constantinescu, E.-M., Sandu, A., Tang, Y., Chai, T., Carmichael, G.-R., Byun, D., and Olaguer, E., 2008. "An adjoint sensitivity analysis and 4d-var data assimilation study of texas air quality". Atmospheric Environment, 42, pp. 5787-5804.

[4] Kang, D., and Lansey, K., 2009. "Real-time demand estimation and confident limit analysis for water distribution systems". Journal of Hydraulic Engineering, 135(10), pp. 825-837.

[5] Waeytens, J., Chatellier, P., and Bourquin, F., 2013. "Sensitivity of inverse advection-diffusionPaper number: FE-16-1416, Corresponding author: Waeytens, Page 17 
Copyright (c) 201 reaction to sensor and control: a low computational cost tool". Computers and Mathematics with Applications, in press.

[6] Lions, J.-L., 1971. Optimal control of systems governed by partial differential equations. Springer.

[7] Wacholder, E., and Dayan, J., 1984. "Application of the adjoint sensitivity method to the analysis of a supersonic ejector". ASME Journal of Fluids Engineering, 106(4), pp. 425-429.

[8] Becker, R., and Rannacher, R., 2001. "An optimal control approach to a posteriori error estimation in finite elements methods". Acta Numerica, Cambridge Press, 10, pp. 1-102.

[9] Waeytens, J., Chamoin, L., and Ladeveze, P., 2012. "Guaranteed error bounds on pointwise quantities of interest for transient viscodynamics problems”. Computational Mechanics, 49(3), pp. 291307.

[10] Chamoin, L., Ladeveze, P., and Waeytens, J., 2014. “Goal-oriented updating of mechanical models using the adjoint framework". Computational Mechanics, 54(6), pp. 1415-1430.

[11] Jameson, A., 1988. "Aerodynamic design via control theory". Journal of Scientific Computing, 3, p. $17 \mathrm{p}$.

[12] Lei, J., and He, J., 2015. “Adjoint-based aerodynamic shape optimization for low reynolds number airfoils". ASME Journal of Fluids Engineering, 138.

[13] E.-M. Papoutsis-Kiachagias, K.-C. G., 2016. “Continuous adjoint methods for turbulent flows, applied to shape and topology optimization: Industrial applications”. Archives of Computational Methods in Engineering, 23(2), pp. 255-299.

[14] Munavalli, G., and Mohan Kumar, M., 2003. "Water quality parameter estimation in a steady state distribution system”. Journal of Water Resources Planning and Management, 192(2), pp. 124-134.

[15] Fabrie, P., Gancel, G., Mortazavi, I., and Piller, O., 2010. "Quality modeling of water distribution systems using sensitivity equations". Hydraulic Engineering, 136(1), pp. 34-44.

[16] Ito, K., and Ravindran, S.-S., 1998. "A reduced-order method for simulation and control of fluid flows". Journal of Computational Physics, 143, pp. 403-425.

[17] Prud'homme, C., Rovas, D., Veroy, K., Machiels, L., Maday, Y., Patera, A. T., and Turinici, G., 2002. "Reliable real-time solution of parametrized partial differential equations: Reduced basis output bound methods". ASME Journal of Fluids Engineering, 124, pp. 70-80.

[18] Besem, F.-M., Kamrass, J.-D., Thomas, J.-P., Tang, D., and Kielb, R.-E., 2016. "Vortex-induced 
Copyright (c) 201 vibration and frequency lock-in of airfoil at high angles of attack". ASME Journal of Fluids Engineering, 138, pp. 1415-1430.

[19] Patnaik, G., Boris, J., Young, T., and Grinstein, F., 2007. "Large scale urban contaminant transport simulations with miles". ASME Journal of Fluids Engineering, 129, pp. 1524-1532.

[20] Rossman, L., 2000. EPANET users' manual. U.S. Environmental Protection Agency, National Risk Management Research Laboratory, Office of Research and Development, Cincinnati.

[21] Blokker, E., Vreeburg, J., Buchberger, S., and van Dijk, J., 2008. "Importance of demand modelling in network water quality models: a review". Drinking Water Engineering and Science Discussions, 1, pp. 1-20.

[22] Formaggia, L., Gerbeau, J.-F., Nobile, F., and Quarteroni, A., 2001. "On the coupling of 3d and $1 \mathrm{~d}$ navier-stokes equations for flow problems in compliant vessels". Computer Methods in Applied Mechanics and Engineering, 191, pp. 561-582.

[23] Jonkergouw, P., Khu, S., Kapelan, Z., and Savic, D., 2008. "Water quality model calibration under unknown demands". Water Resources Planning and Management, pp. 326-336.

[24] Vreeburg, J., and Boxall, J., 2007. "Discoulouration in potable water distribution systems: a review". Water Research, 41, 519-529.

[25] Hecht, F., 2012. "New development in freefem++". J. Numer. Math., 20(3-4), pp. 251-265.

[26] Vasconselos, J., Rossman, L., Grayman, W., Boulos, P., and Robert, M., 1997. "Kinetics of chlorine decay". Journal - American Water Works Association, 89(7), pp. 54-65.

[27] Wu, Z., and Chen, G., 2014. "Approach to transverse uniformity of concentration distribution of a solute in a solvent flowing along a straight pipe". Journal of Fluid Mechanics, 740, pp. 196-213.

[28] Pironneau, O., Liou, J., and Tezduyar, T. T. . I., 1992. "Characteristic galerkin and galerkin least squares space-time formulations for the advection-diffusion equation with time dependent domain”. Computer Methods in Applied Mechanics and Engineering, 100, pp. 117-141.

[29] Rossman, L. A., Clark, R. M., and Grayman, W. M., 1994. "Modeling chlorine residuals in drinking-water distribution systems”. Journal of Environnemental Engineering, 120(4), pp. 803820.

[30] Morozov, V. A., 1966. "On the solution of functional equations by the method of regularization". Soviet Math. Dokl., 7, pp. 414-417. 
Copyright [31] Munavalit, G., and Mohan Kumar, M., 2005. "Water quality parameter estimation in a distribution system under dynamic state". Water Research, 39, pp. 4287-4298.

Paper number: FE-16-1416, Corresponding author: Waeytens, Page 20 


\section{Copyright List of Figures}

1 2D study case geometry and sensor placement (dimensions in $\mathrm{mm}$ )

2 Temporal evolution of $\left(v_{c_{1}}^{\max }\right)$ and $\left(v_{c_{3}}^{\max }\right)-$

3 Temporal evolution of $\left(u_{1}\right)_{e x}$ and $\left(u_{3}\right)_{e x}$

$4 \quad$ Fine mesh - $2.8 \times 10^{4}$ degree of freedom (dof)

5 Coarse mesh $-1.9 \times 10^{3}$ degree of freedom (dof)

6 Influence of flow uncertainty on the chlorine reconstruction - Sensors $1 \& 2$ - Fine discretization $\left(2.8 \times 10^{4}\right.$ dof 1280 time steps $)$ for chlorine concentration

$\varepsilon_{C}$ represents the $\mathrm{L} 2$ error between the exact concentration and the reconstructed concentration; $n_{i t}$ is the number of iterations of the inverse problem

7 Influence of the flow model error and the flow discretization error on the chlorine reconstruction - Sensors $1 \& 2$ - Fine discretization $\left(2.8 \times 10^{4}\right.$ dof 1280 time steps $)$ for chlorine concentration

$\varepsilon_{C}$ represents the $\mathrm{L} 2$ error between the exact concentration and the reconstructed concentration; $\varepsilon_{v}$ represents the $\mathrm{L} 2$ error between the exact velocity and the approximate velocity; $n_{i t}$ is the number of iterations of the inverse problem

8 Norm of the approximate velocities $\left\|v_{h}\right\|$ at $t=T / 2$, which are obtained using sophisticated or simple flow models in the velocity reconstruction process - Fine mesh $\left(6.4 \times 10^{4}\right.$ dof 256 time steps)

9 Reference concentration at $t=3 T / 5$ when considering reference velocity obtained with a very fine discretization - Concentration discretization $\left(1.1 \times 10^{5}\right.$ dof 2560 time steps $)$

10 Reconstructed concentration at $t=3 T / 5$ when considering approximate velocities obtained from the velocity reconstruction process - Sensors $1 \& 2$ - Concentration Fine discretization $\left(2.8 \times 10^{4}\right.$ dof 1280 time steps $)$

Paper number: FE-16-1416, Corresponding author: Waeytens, Page 21 
Copyright (c) $2911^{7}$ by Infiuence of flow model error and flow discretization error on the chlorine reconstruction - Sensors $1 \& 2$ - Coarse discretization $\left(1.9 \times 10^{3}\right.$ dof 256 time steps $)$ for chlorine concentration

$\varepsilon_{C}$ represents the $\mathrm{L} 2$ error between the exact concentration and the reconstructed concentration; $\varepsilon_{v}$ represents the L2 error between the exact velocity and the approximate velocity; $n_{i t}$ is the number of iterations of the inverse problem

12 Fluctuating boundary velocity

13 Influence of flow model error and flow discretization error on the chlorine reconstruction - Sensors $1 \& 2$ - Coarse discretization $\left(1.9 \times 10^{3}\right.$ dof 256 time steps $)$ for chlorine concentration - Representative flow in "distribution mains"

$\varepsilon_{C}$ represents the L2 error between the exact concentration and the reconstructed concentration; $\varepsilon_{v}$ represents the L2 error between the exact velocity and the approximate velocity; $n_{i t}$ is the number of iterations of the inverse problem

14 Sensor outputs with noise $\mathscr{N}\left(0, \sigma^{2}\right)$ : sensor $S_{1}$ on the left and sensor $S_{2}$ on the right

15 Influence of the noise variance $\sigma^{2}$ on the chlorine reconstruction - Sensor $1 \& 2$ - Fine discretization $\left(2.8 \times 10^{4}\right.$ dof, 1280 time steps $)$

16 Influence of the noise normal distribution mean $\mu$ on the chlorine reconstruction - Sensor $1 \& 2$ - Fine discretization $\left(2.8 \times 10^{4}\right.$ dof, 1280 time steps $)$

\section{List of Tables}

1 Chlorine reconstruction for the Navier-Stokes flow - Sensors $1 \& 2$ - Fine mesh $(2.8 \times$ $10^{4}$ dof 1280 time steps) - High kinetic coefficient: $k=5 d a y^{-1} \ldots \ldots$

2 Chlorine reconstruction for the Navier-Stokes flow - Sensors $1 \& 2$ - Coarse mesh $(1.9 \times$ $10^{3}$ dof 256 time steps) - High kinetic coefficient: $k=5 d a y^{-1} \ldots \ldots$

3 Chlorine reconstruction for the Navier-Stokes flow - Sensors $1 \& 2$ - Fine mesh $(2.8 \times$ $10^{4}$ dof 1280 time steps) - Low kinetic coefficient: $k=0.5 d a y^{-1} \ldots \ldots$ 


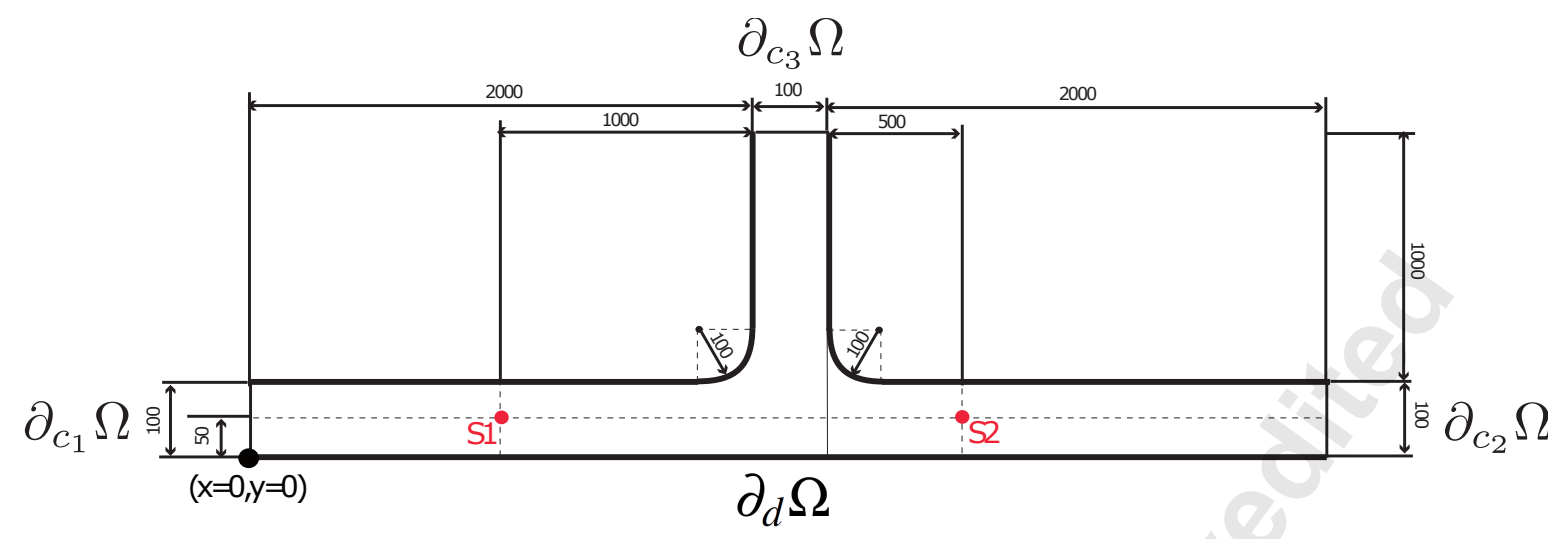

Fig. 1. 2D study case geometry and sensor placement (dimensions in $\mathrm{mm}$ )

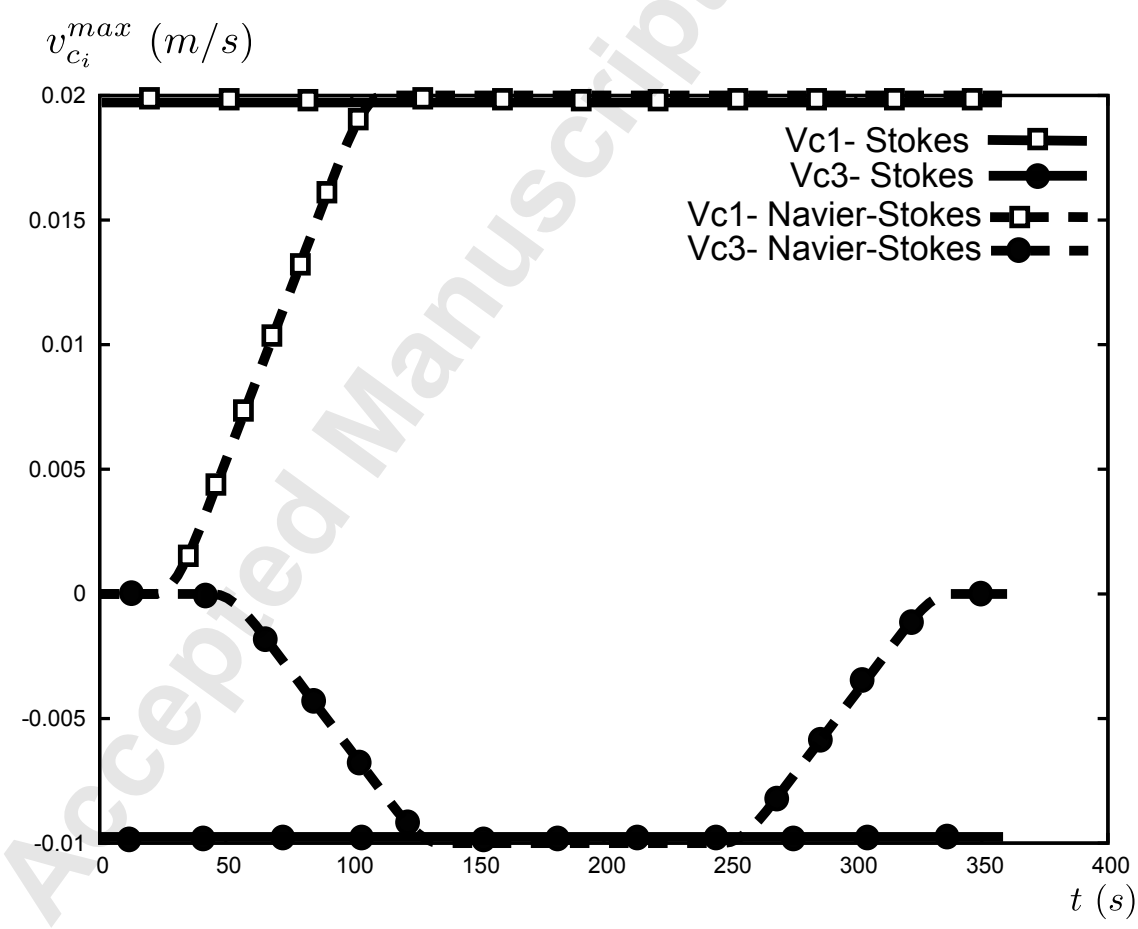

Fig. 2. Temporal evolution of $\left(v_{c_{1}}^{\max }\right)$ and $\left(v_{c_{3}}^{\max }\right)$ -

Paper number: FE-16-1416, Corresponding author: Waeytens, Page 23 


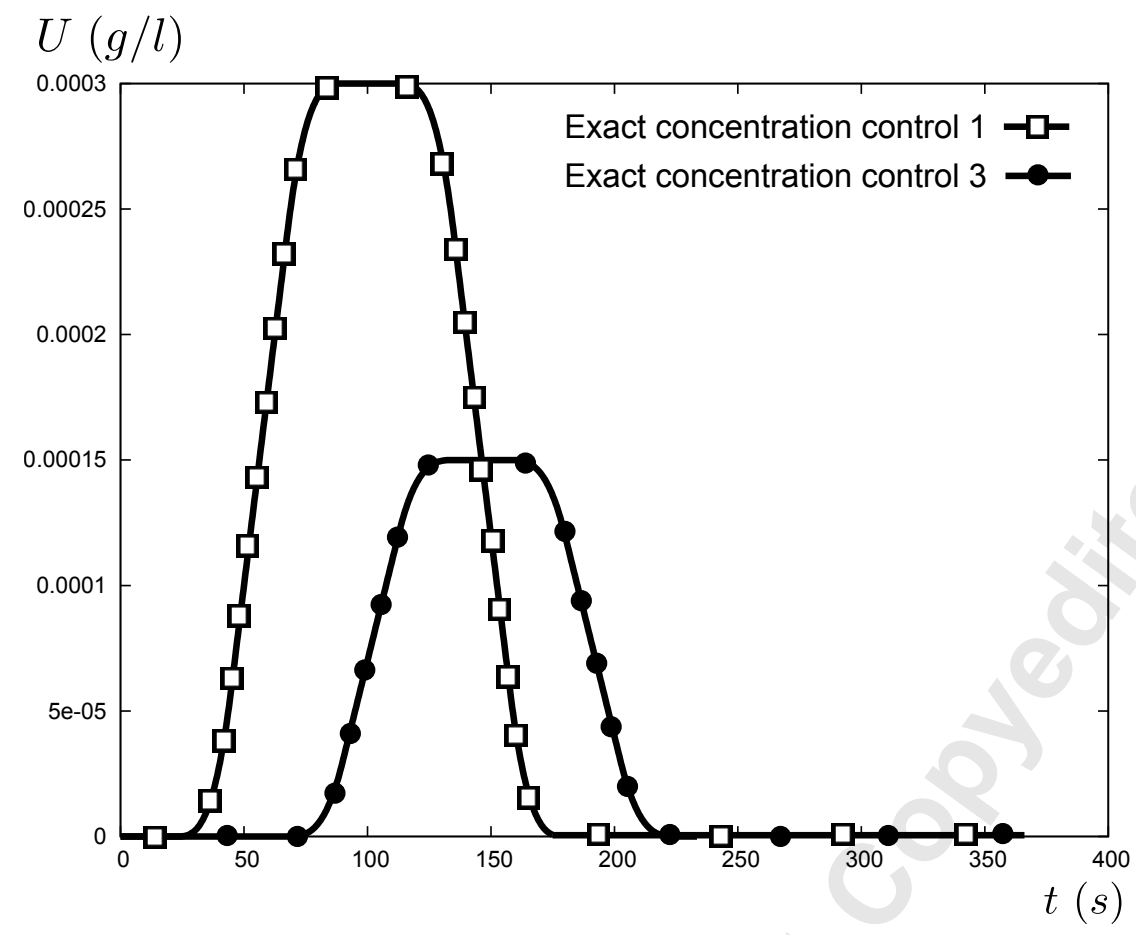

Fig. 3. Temporal evolution of $\left(u_{1}\right)_{e x}$ and $\left(u_{3}\right)_{e x}$

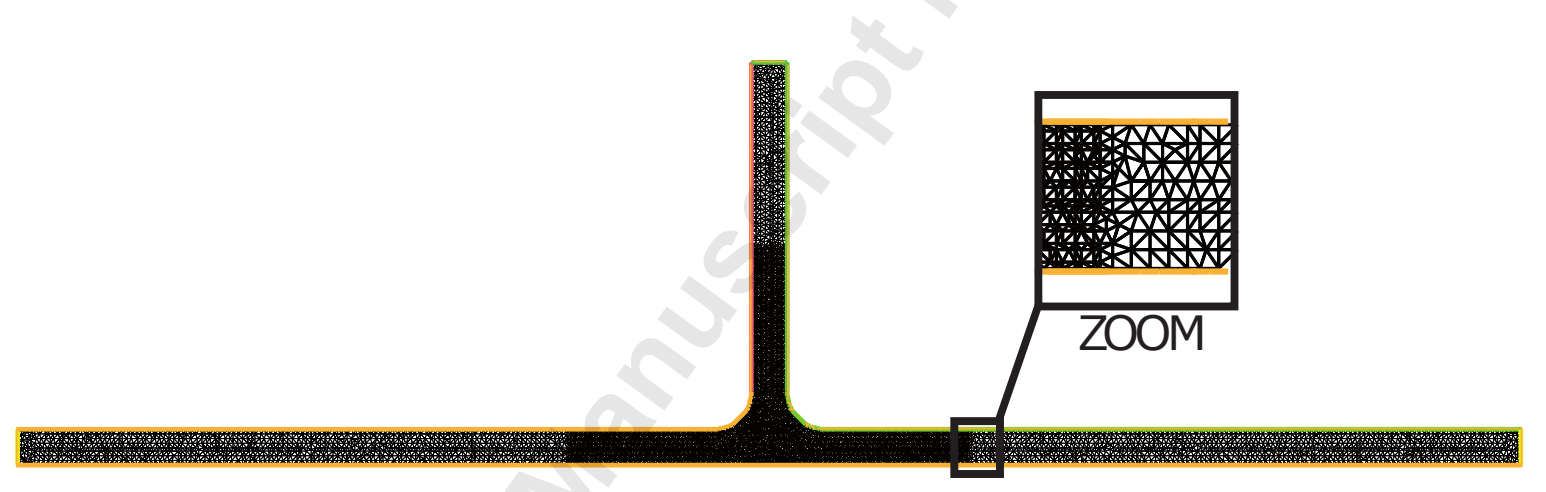

Fig. 4. Fine mesh $-2.8 \times 10^{4}$ degree of freedom (dof)

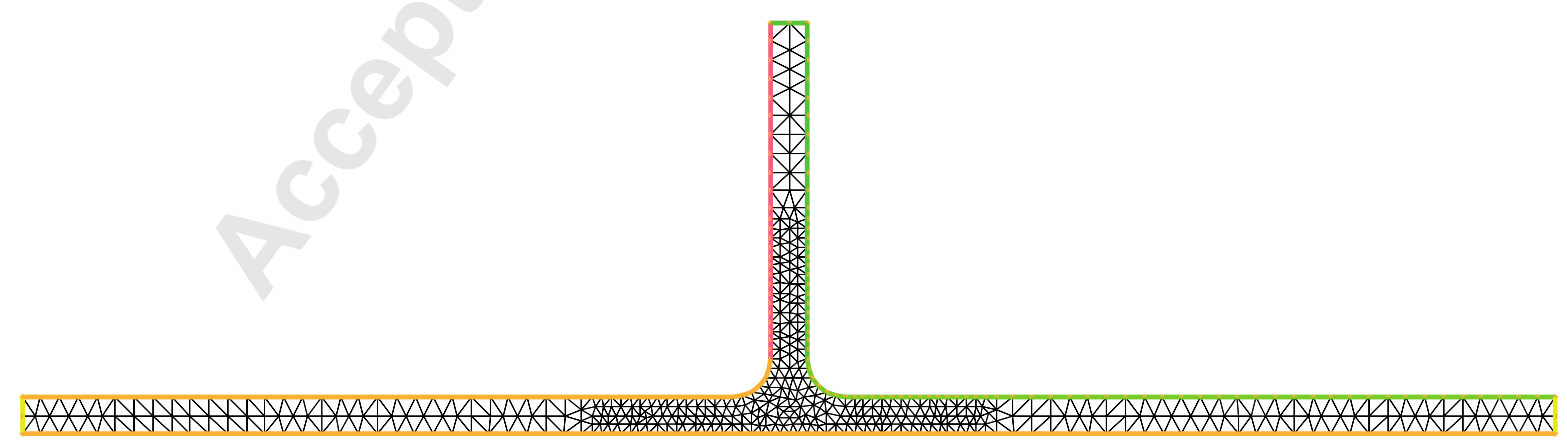

Fig. 5. Coarse mesh $-1.9 \times 10^{3}$ degree of freedom (dof)

Paper number: FE-16-1416, Corresponding author: Waeytens, Page 24 


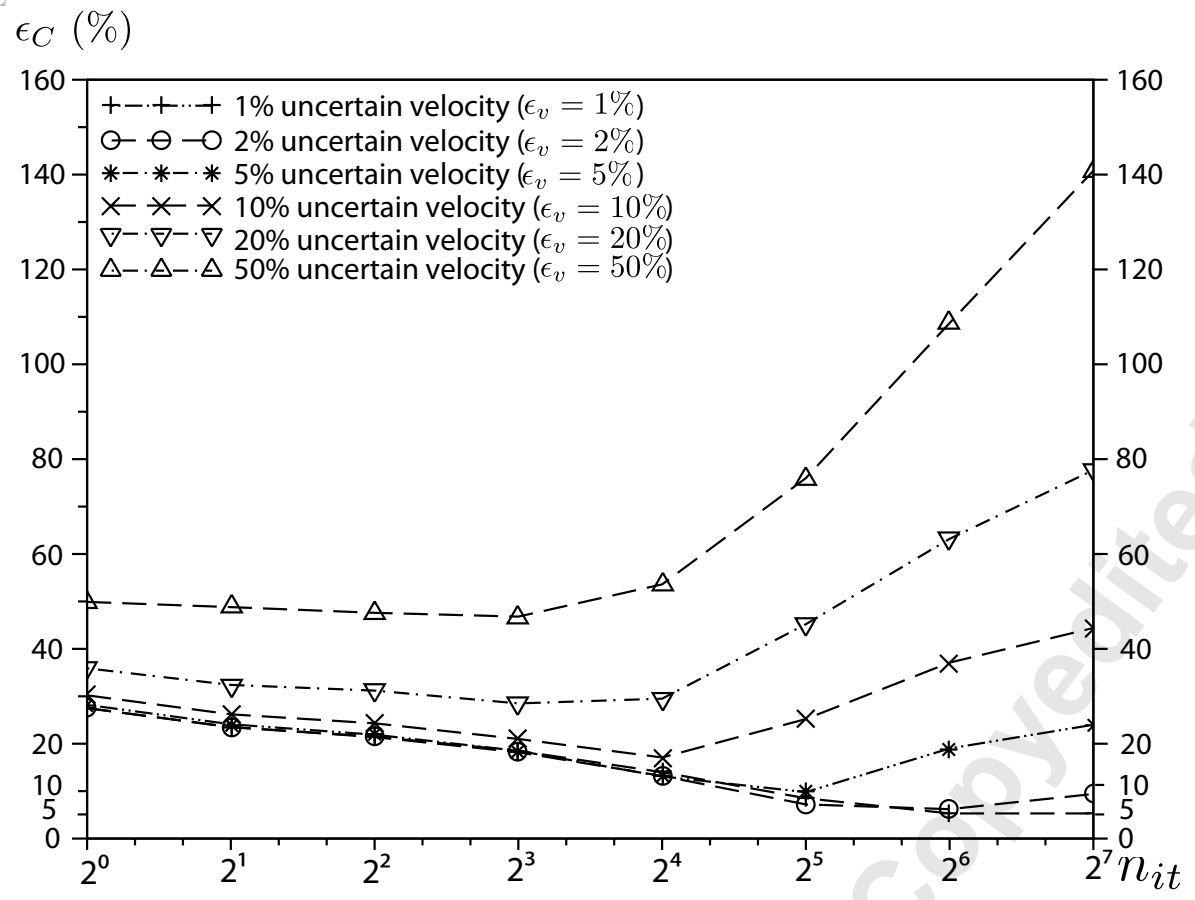

Fig. 6. Influence of flow uncertainty on the chlorine reconstruction - Sensors 1 \& 2 - Fine discretization $\left(2.8 \times 10^{4}\right.$ dof 1280 time steps $)$ for chlorine concentration

$\varepsilon_{C}$ represents the $\mathrm{L} 2$ error between the exact concentration and the reconstructed concentration; $n_{i t}$ is the number of iterations of the inverse problem

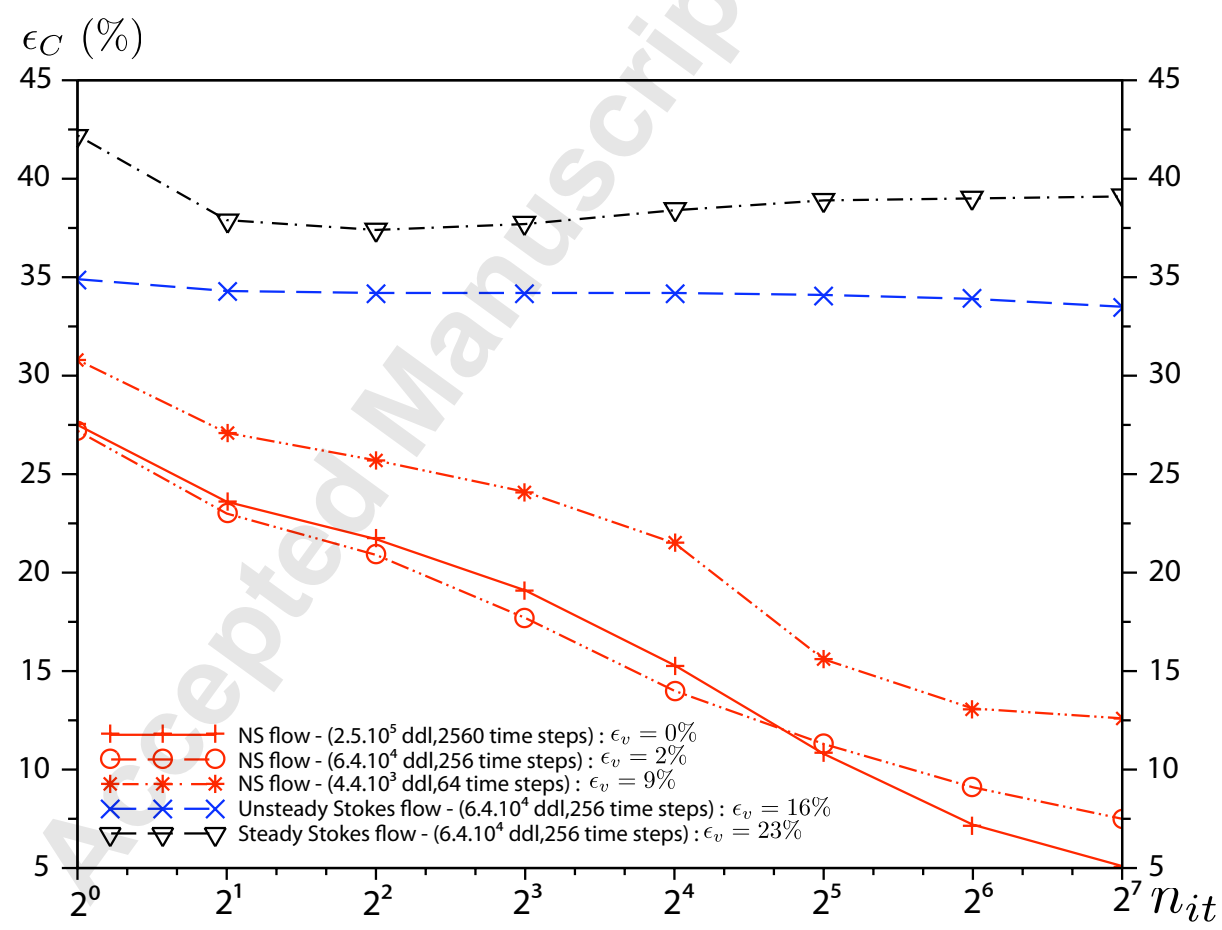

Fig. 7. Influence of the flow model error and the flow discretization error on the chlorine reconstruction - Sensors 1 \& 2 - Fine discretization $\left(2.8 \times 10^{4}\right.$ dof 1280 time steps) for chlorine concentration

$\varepsilon_{C}$ represents the $\mathrm{L} 2$ error between the exact concentration and the reconstructed concentration; $\varepsilon_{v}$ represents the L2 error between the exact velocity and the approximate velocity; $n_{i t}$ is the number of iterations of the inverse problem

Paper number: FE-16-1416, Corresponding author: Waeytens, Page 25 

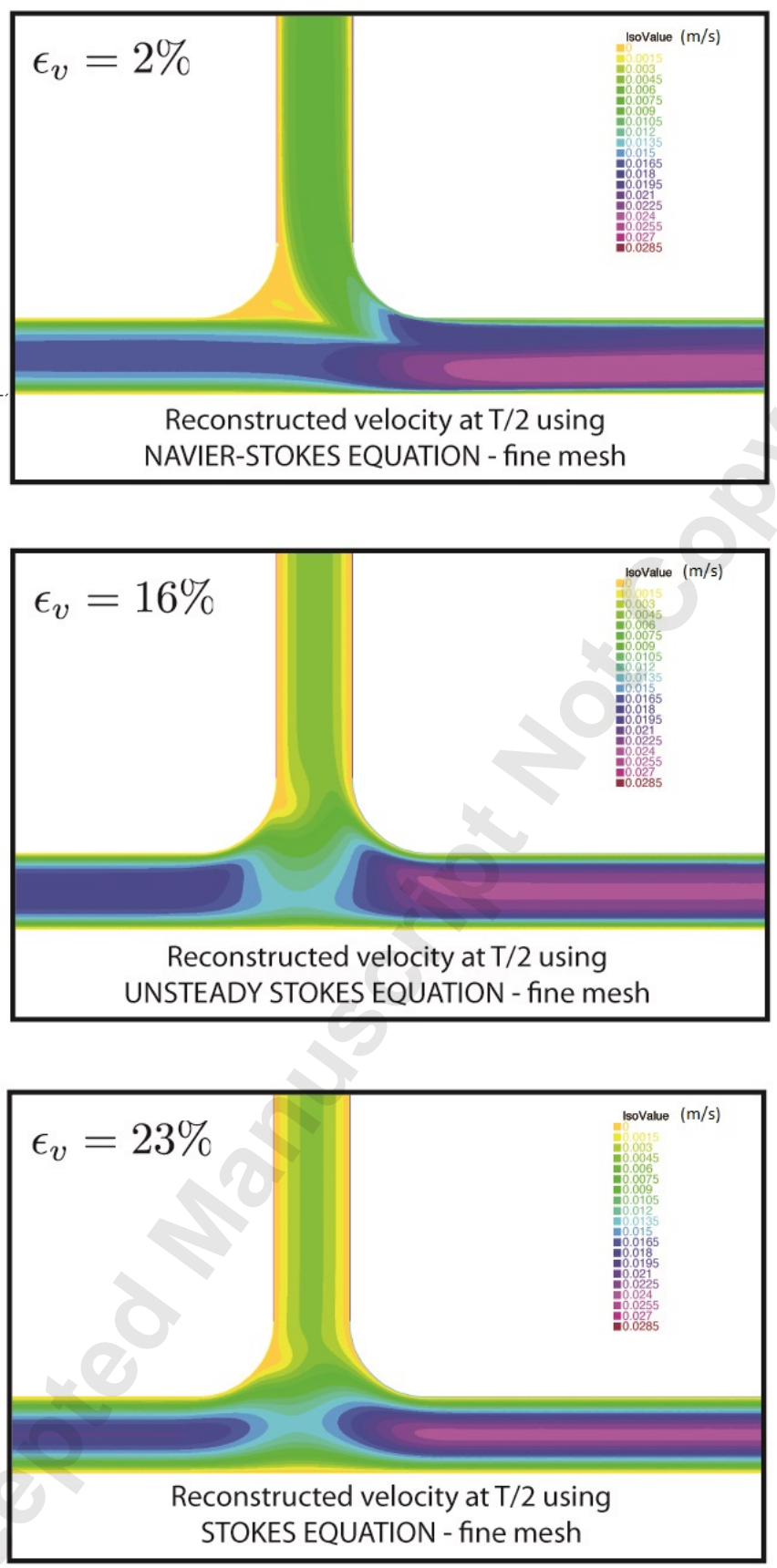

Fig. 8. Norm of the approximate velocities $\left\|v_{h}\right\|$ at $t=T / 2$, which are obtained using sophisticated or simple flow models in the velocity reconstruction process - Fine mesh $\left(6.4 \times 10^{4}\right.$ dof 256 time steps)

Paper number: FE-16-1416, Corresponding author: Waeytens, Page 26 


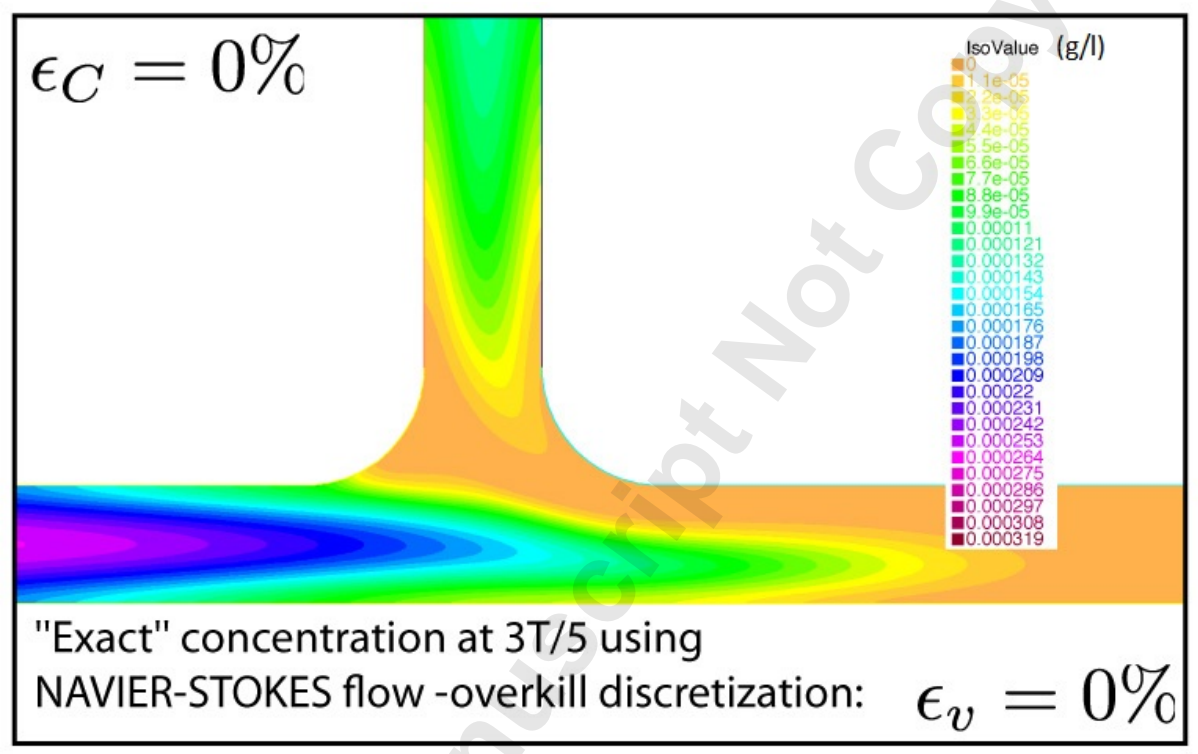

Fig. 9. Reference concentration at $t=3 T / 5$ when considering reference velocity obtained with a very fine discretization - Concentration discretization $\left(1.1 \times 10^{5}\right.$ dof 2560 time steps)

Paper number: FE-16-1416, Corresponding author: Waeytens, Page 27 

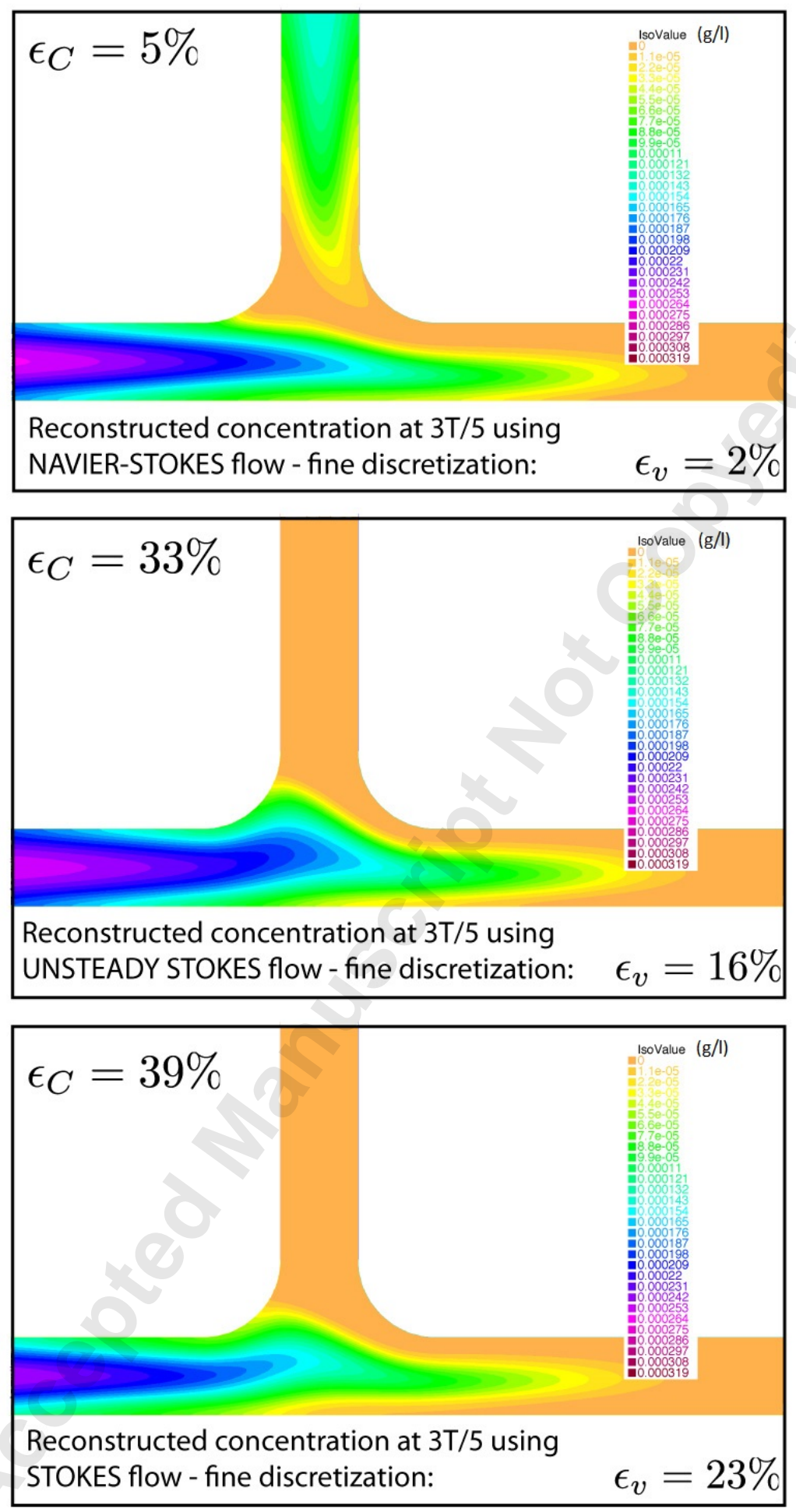

Fig. 10. Reconstructed concentration at $t=3 T / 5$ when considering approximate velocities obtained from the velocity reconstruction process - Sensors $1 \& 2$ - Concentration Fine discretization $\left(2.8 \times 10^{4}\right.$ dof 1280 time steps $)$

Paper number: FE-16-1416, Corresponding author: Waeytens, Page 28 


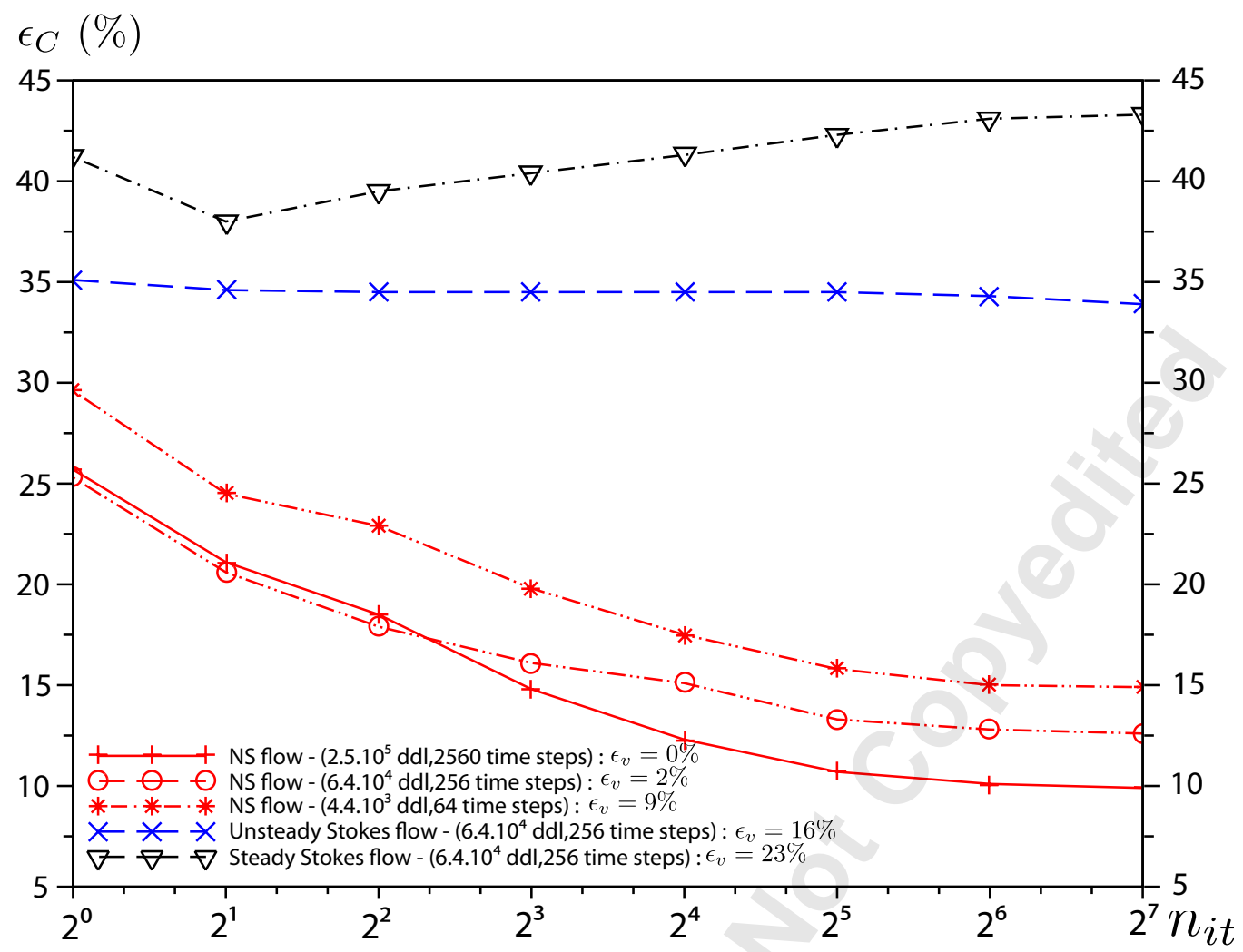

Fig. 11. Influence of flow model error and flow discretization error on the chlorine reconstruction - Sensors 1 \& 2 - Coarse discretization $\left(1.9 \times 10^{3}\right.$ dof 256 time steps) for chlorine concentration

$\varepsilon_{C}$ represents the $\mathrm{L} 2$ error between the exact concentration and the reconstructed concentration; $\mathcal{E}_{v}$ represents the L2 error between the exact velocity and the approximate velocity; $n_{i t}$ is the number of iterations of the inverse problem
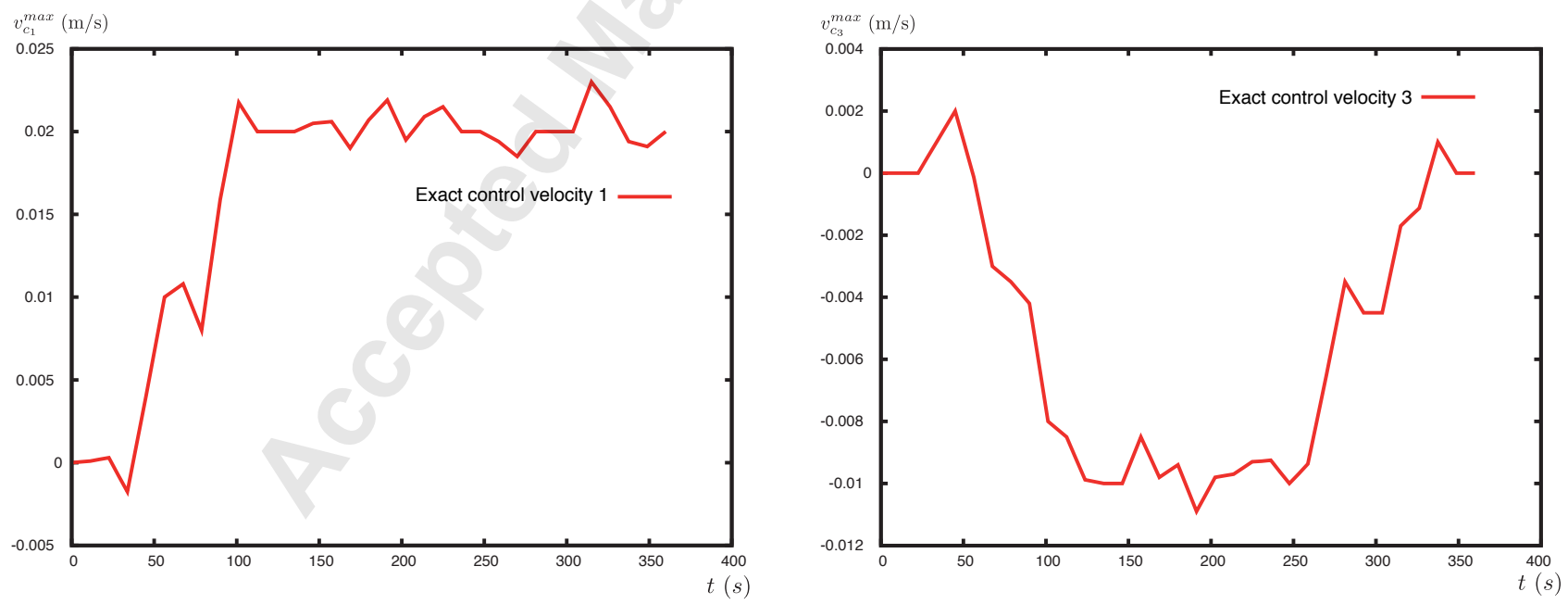

Fig. 12. Fluctuating boundary velocity

Paper number: FE-16-1416, Corresponding author: Waeytens, Page 29 


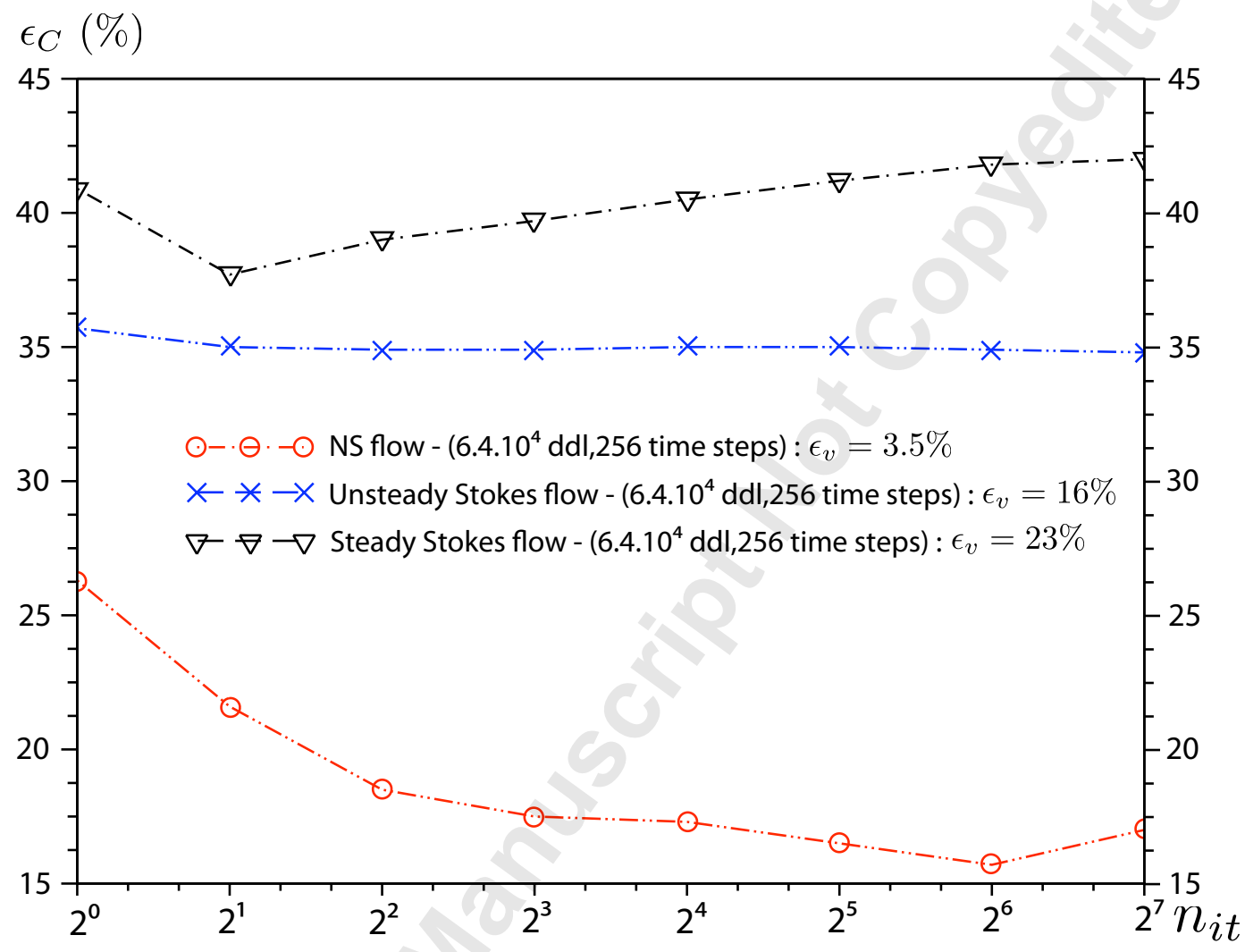

Fig. 13. Influence of flow model error and flow discretization error on the chlorine reconstruction - Sensors 1 \& 2 - Coarse discretization $\left(1.9 \times 10^{3}\right.$ dof 256 time steps) for chlorine concentration - Representative flow in "distribution mains"

$\varepsilon_{C}$ represents the $\mathrm{L} 2$ error between the exact concentration and the reconstructed concentration; $\mathcal{E}_{v}$ represents the L2 error between the exact velocity and the approximate velocity; $n_{i t}$ is the number of iterations of the inverse problem

Paper number: FE-16-1416, Corresponding author: Waeytens, Page 30 

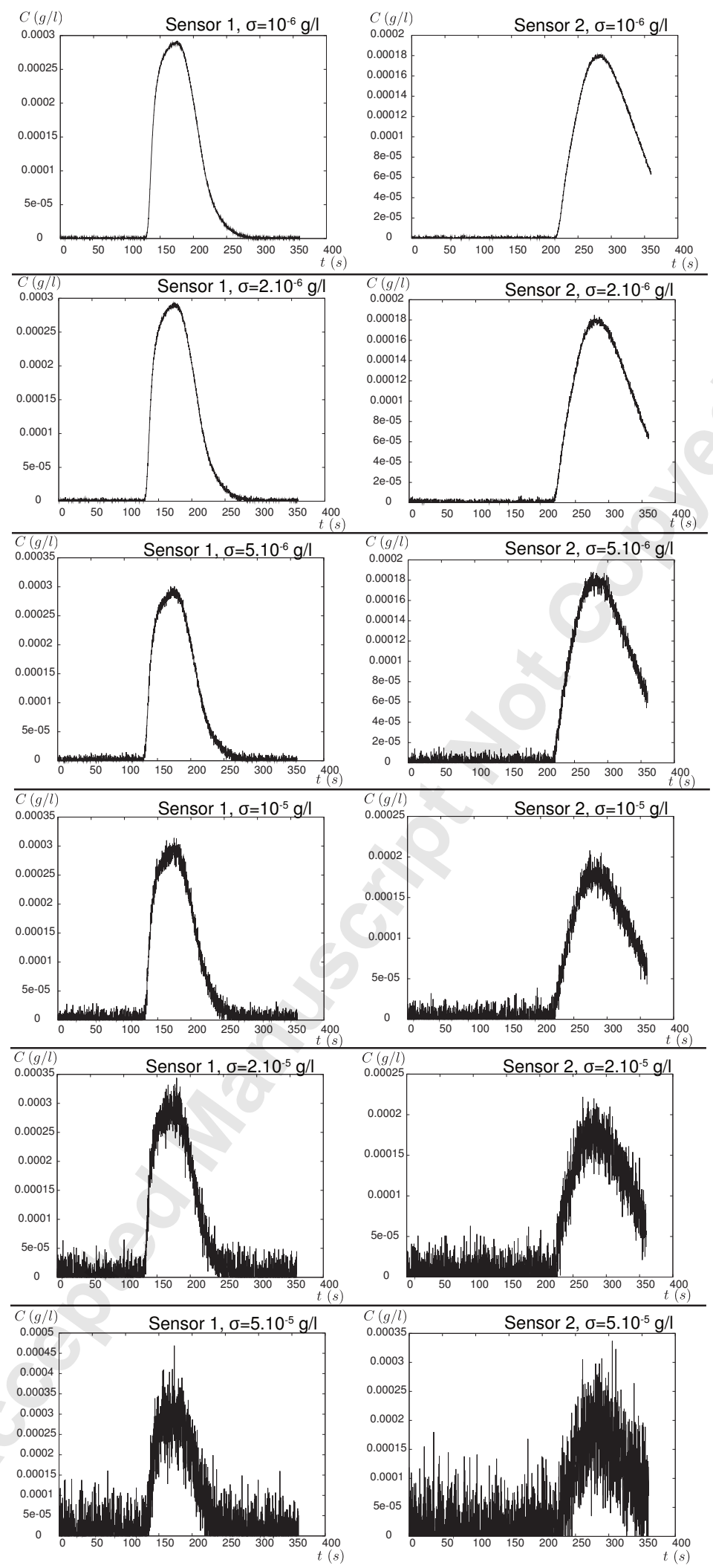

Fig. 14. Sensor outputs with noise $\mathscr{N}\left(0, \sigma^{2}\right)$ : sensor $S_{1}$ on the left and sensor $S_{2}$ on the right

Paper number: FE-16-1416, Corresponding author: Waeytens, Page 31 


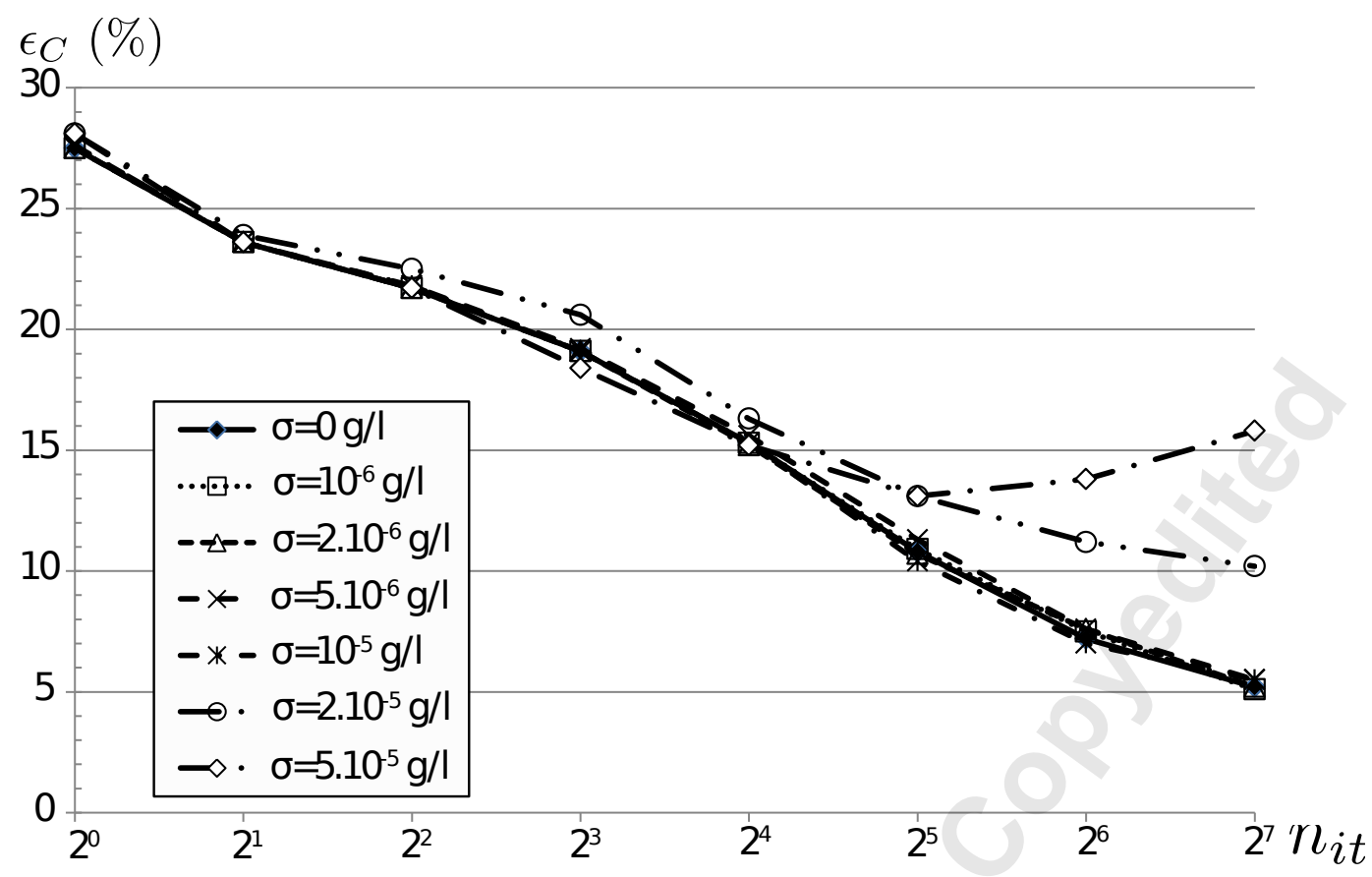

Fig. 15. Influence of the noise variance $\sigma^{2}$ on the chlorine reconstruction - Sensor $1 \& 2$ - Fine discretization $\left(2.8 \times 10^{4}\right.$ dof, 1280 time steps)

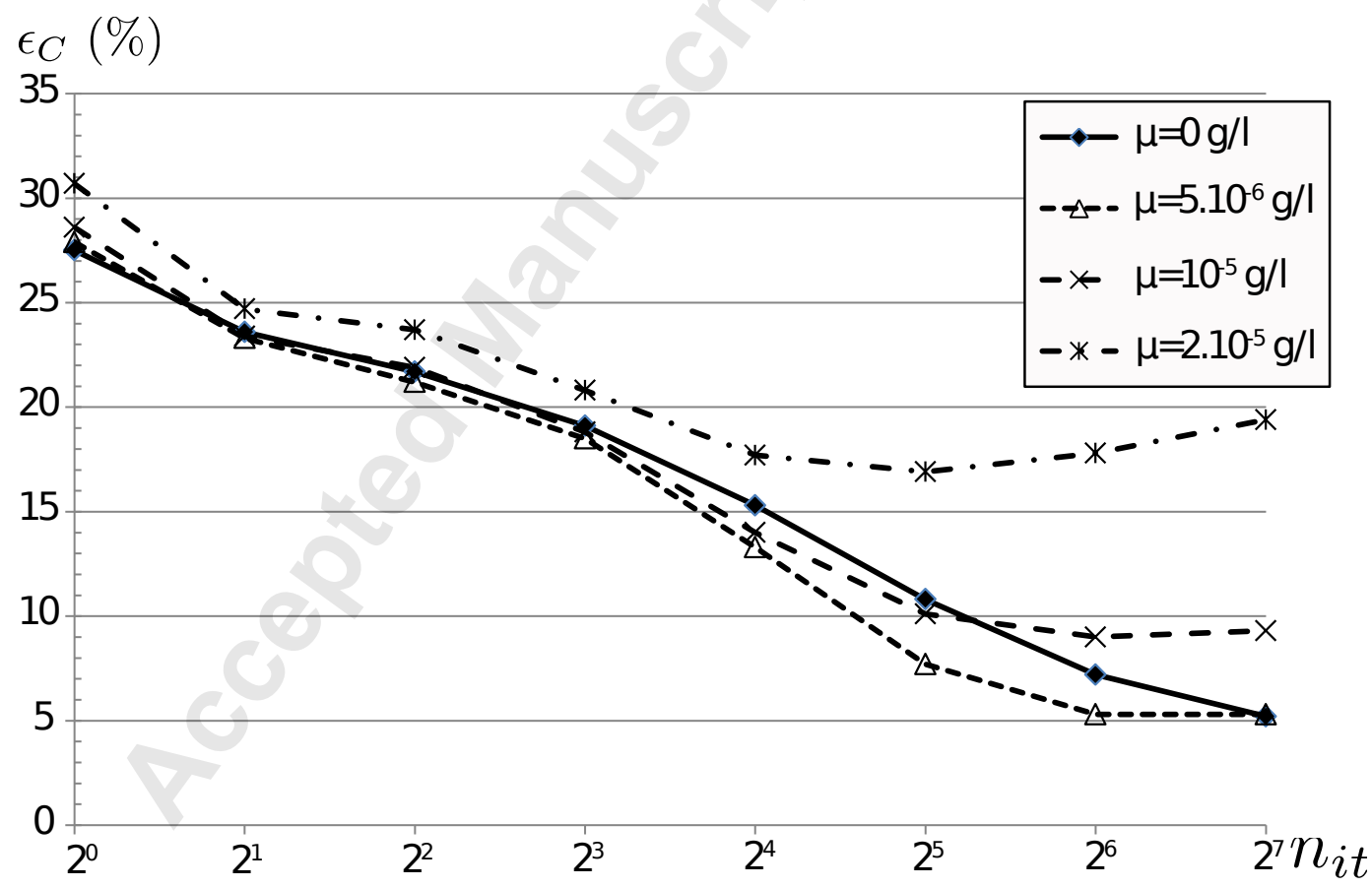

Fig. 16. Influence of the noise normal distribution mean $\mu$ on the chlorine reconstruction - Sensor $1 \& 2-$ Fine discretization $\left(2.8 \times 10^{4}\right.$ dof, 1280 time steps)

Paper number: FE-16-1416, Corresponding author: Waeytens, Page 32 